MPIKG Public Access

Author Manuscript

Published in final edited form as:

Radziuk, D. V., \& Möhwald, H. (2016). Ultrasonic Mastering of Filter Flow and Antifouling of

Renewable Resources. ChemPhysChem, 17(7), 931-953. doi:10.1002/cphc.201500960.

\title{
Ultrasonic Mastering of Filter Flow and Antifouling of Renewable Resources
}

Darya Radziuk* and Helmuth Möhwald

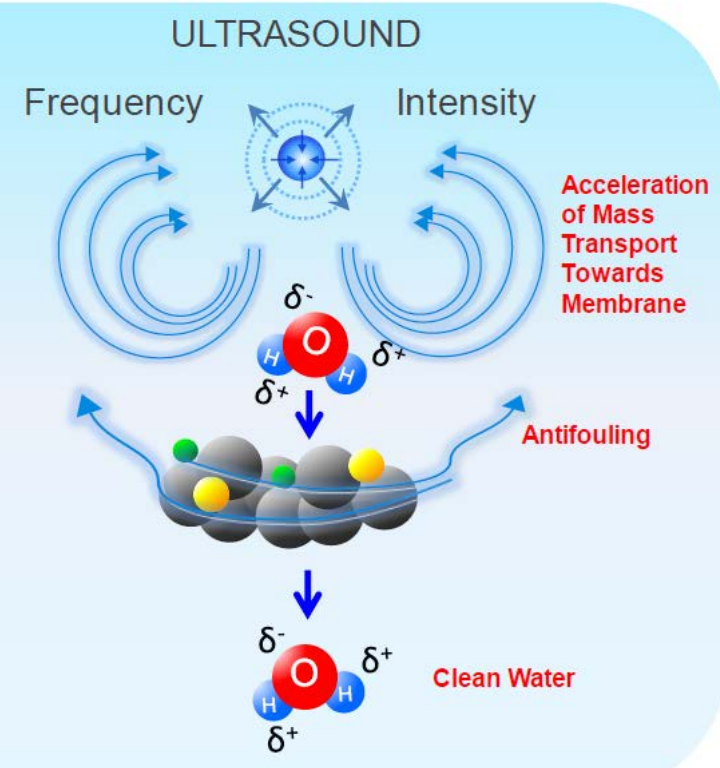

This article may be used for non-commercial purposes in accordance with Wiley Terms and Conditions for Self-Archiving. 


\section{Abstract:}

Inadequate access to purify water and sanitation requires new ergonomic methods at lower cost, less energy consumption, minimal use of chemicals and impact on the environment. Among them ultrasound is a unique means to control physics and chemistry of complex fluid (wastewater) with excellent performance in mass transfer, cleaning and disinfection. In membrane filtration processes it overcomes diffusion limits and can accelerate the fluid flow towards the filter preventing antifouling. Here we outline the current state of knowledge and technological design with the focus on physico-chemical strategies of ultrasound for water cleaning. We highlight important parameters of ultrasound for the delivery of fluid flow from a technical perspective employing principles of physics and chemistry. By introducing various ultrasonic methods involving bubbles or cavitation in combination with external fields we show advancements in flow acceleration and mass transportation to the filter. In most of them we emphasize the main role of streaming and impact of cavitation with a perspective to prevent and remove fouling deposits during the flow. We also elaborate on the deficiencies of present technology and on problems to be solved to achieve a wide spread application.

\section{Introduction}

Industrial wastewater treatment has become a global issue for both developed and developing countries due to high concentration of pollutants, especially refractory organic compounds. ${ }^{[1]}$ Inadequate access to clean water and sanitation is expected to become worse in the coming decades even in regions considered water-rich. As a growing number of contaminants from human activity are entering the water supplies, research efforts are focused to purify water previously considered clean. New methods at lower cost, less energy consumption, minimal use of chemicals and impact on the environment are demanded. Among these methods membrane filtration is widely accepted as a process technology because of its versatility, production of a consistent end product at lower cost with less energy. However, inherent membrane fouling, which is caused by concentration polarization and pore blocking, reduces the permeate flux with operation time and limits its industrial application. Also mass transport in a fluid flow requires new approaches, which could prevent fouling and avoid altering of the membrane structure.

Ultrasound can significantly increase water flux and decrease the solute concentration near membrane filters. With ultrasound no chemical cleaning reagent is required and a high permeate flux can be maintained throughout the filtration process, not only immediately after cleaning. ${ }^{[2]}$ Mass transport and phase transfer can be accelerated by ultrasound. Surface coatings can be ultrasonically removed, modified or renewed at faster speed and lower chemical consumption. Ultrasonic conversion of compounds and their degradation occur with higher yields than by conventional procedures obeying the golden rules of 'green chemistry'. ${ }^{[3]}$ In addition, wastewater contaminants can be oxidized by hydroxyl radicals, pyrolytic decomposition and supercritical water. ${ }^{[4]}$ In water disinfection ultrasound inactivates bacteria through their declustering and radical attack. ${ }^{[5]}$ In this way, ultrasound creates unique physicochemical conditions and can be used as an effective tool to increase the fluid flow, degrade contaminated species and prevent the fouling of filters at the same time.

Dr. Darya Radziuk and Prof. Helmuth Möhwald

Max-Planck Institute of Colloids and Interfaces

Am Mühlenberg 1,

D-14476 Potsdam (Science Park Golm)

Germany

E-mail: darya.radziuk@mpikg.mpg.de 
Helmuth Möhwald received his diploma in physics (1971) and $\mathrm{PhD}$ (1974) from Univ. Gottingen (Germany) for research in organic solids. After working as a postdoc at IBM San Jose, assistant professor at Univ. Ulm, and research scientist at Dornier Systems, he became an associate professor in biophysics at TU Munich (1981). From a chair in physical chemistry

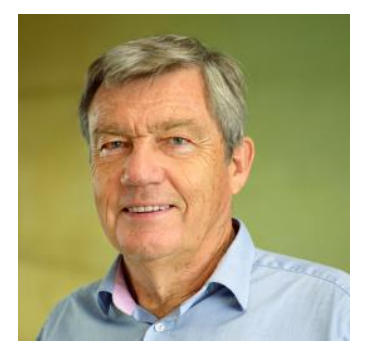
at Univ. Mainz (1987) he became the founding director of the Max Planck Institute of Colloids and Interfaces in Potsdam (1993). His recent honours have been the Overbeek medal, the Gay-Lussac-Humboldt award (2007), an honorary doctorate or Univ. Montpellier (2008) and the WolfgangOstwald award (2009).

Darya Radziuk received her diploma in Physics (2004) from Belarussian State University. After working as research scientist at IT Bevalex Ltd. in Minsk (2005-2006) she joined Max-Planck Institute of Colloids and Interfaces as a researcher (2006) and obtained her PhD in Physics (2007-2010) at the University of Potsdam (Germany). She was awarded the Jean-Louis Luche Prize from the European Society of Sonochemistry (2008), "Best Technical Paper" at the 5th

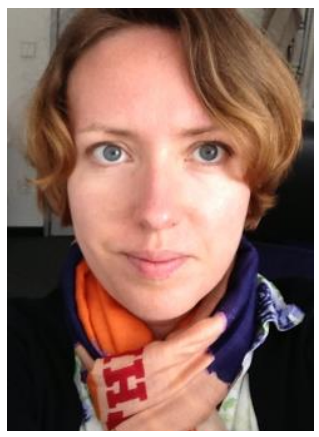
MPA Meeting (2011) and a DFG fellowship (2011) while working at the MaxPlanck Institute of Colloids and Interfaces. After joining the group of Prof. K. Suslick as a postdoc fellow (2011) she returned to Max-Planck Institute of Colloids and Interfaces (2013). Her main scientific interests concern ultrasound and acoustic cavitation, sonoluminescence, sensors, interfacial sonochemistry and physico-chemical processes of complex liquid/liquid interfaces.

As a longitudinal wave of periodic compression/rarefaction cycles ultrasound can transport fluid flows in a minimal invasive manner at lower cost and faster speed (Scheme 1). This fluid transport can be advanced by the type of acoustic cavitation, i.e. stable or transient. In a stable cavitation bubble oscillation forms streaming flows and accelerates the mass transport avoiding damage of a nearby surface. Such cavitation bubbles do not implosively collapse and can be formed at higher frequency of ultrasound ( $>$ hundreds $\mathrm{kHz}$ or $\mathrm{MHz}$ ). In contrast, transient cavitation requires lower frequency of ultrasound ( $<$ hundreds $\mathrm{kHz}$ ) and violent bubble collapse can form intense shock waves or jets $(\mu \mathrm{m})$. A typical shock wave scenario occurs within ns in a symmetrically collapsing bubble due to adiabatic heating of a gas $(1600 \mathrm{~K}-9000 \mathrm{~K})$. In contrast, cooler liquid jets (> $100 \mathrm{~m} / \mathrm{s}$ ) arise due to the asymmetry from a disturbing boundary Streaming under these conditions becomes stronger and forms turbulent flows with higher gradients of pressure $(\Delta P)$ and temperature $(\Delta T)$. It is the radiation force of streaming ${ }_{,}^{[6]}$ which can disrupt the intermolecular forces and transport the substances in a fluid flow of filters. Its strength depends on the intensity of the acoustic field (energy input) and the frequency of ultrasound (energy output).

In general, most of the membrane filters face the problem of slow fluid flow due to weak diffusion. Transient acoustic cavitation can create effective diffusion coefficients with at least 10 -fold magnitude higher due to streaming and turbulence. ${ }^{[7]}$ At the same time it can prevent the fouling due to interparticle contacts through their mutual motion. The selectivity of accelerated fluid flow can be achieved in the presence of an electric field $(\Delta E)$. Mass transport to the membrane can be also accelerated as a result of faster streaming in complex interaction between shock waves and jets during turbulence (Scheme 1).

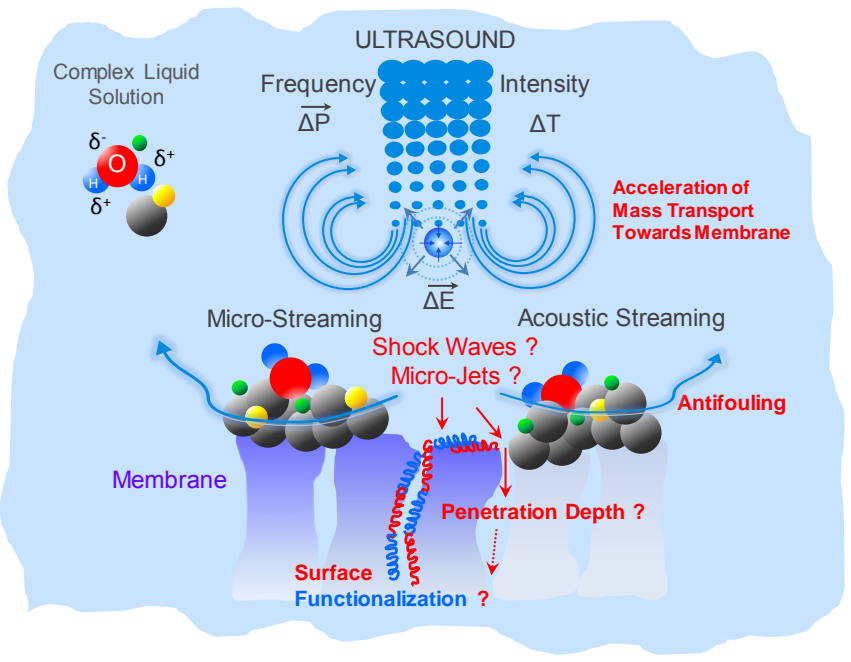

Scheme 1. Schematic illustration of the interaction between ultrasound and a membrane through acoustic cavitation in complex liquid solution (wastewater). In a liquid solution ultrasound propagates as a pressure wave with compression/rarefaction cycles and forms acoustic cavitation. This can be defined as bubble formation, growth and collapse with emanating energy (high pressure and temperature) in the bulk solution. The energy of the bubble collapse can be controlled via the frequency and intensity of acoustic pressure field created by ultrasound. As the bubble energy upon collapse is high, larger pressure $(\Delta \mathrm{P})$ and temperature $(\Delta \mathrm{T})$ gradients can be produced in a liquid. These create turbulence and streaming of fluid flows and can accelerate mass transport towards the membrane surface. It is suggested that $\mu$-streaming and acoustic streaming can be the main mechanisms in membrane antifouling, i.e. removal of a cake or gel-like layer containing particulate impurities. The antifouling by acoustic means can be significantly improved in the presence of electric field gradients $(\Delta \mathrm{E})$. Intuitively $\mu$-jets may also take an active part in the membrane antifouling, but this is not understood yet especially in relation to the surface chemistry of the membrane and the penetration depth.

At present still in bubble clouds the impact of shock waves and jets cannot be distinguished. The dynamic fluid transport across the membrane filter can be another difficult problem after antifouling (Scheme 1). Most of the studies reveal the technical difficulty of cleaning of inner filtration pores and ultrasound is not an exception. ${ }^{[8]}$ Theoretically liquid jets could penetrate the inner 
pores, but its technical realization would require appropriate jetlengths to reach the penetration depth and surface functionalization of the filter structure (Scheme 1). As the structure of liquid jets depends on the distance from a disturbing boundary (among other factors), absorption and reflection (also scattering) of ultrasound should take an important part. Other critical parameters of ultrasound are its frequency and intensity, viscosity of fluid and viscoelastic properties of a membrane filter and its interfacial topology.

In this review, we take a look at ultrasound as a unique means to control physics and chemistry of a complex fluid (wastewater) in membrane filters from a technical perspective. At first, we highlight important parameters of ultrasound to overcome diffusion limits in the delivery of fluid flows to the membrane surface. By introducing various ultrasonic methods involving bubbles or cavitation we show advancements in flow acceleration and mass transportation to the membrane. The versatility of approaches involves the combination of ultrasound with mechanical pumping action, thermal gradients, magnetic stirring, and interaction with the viscoelastic material, entrapment of bubbles in micro-fluidic environment or electric field. In most of these methods we emphasize the main role of streaming as a driving force for accelerated fluid flow and also antifouling. Next, we highlight the impact of ultrasound and cavitation on the morphology and composition of membrane filters with a perspective to prevent and remove fouling deposits during the flow. Overall, here we describe the current state of knowledge and outline technical approaches and physicochemical strategies of fluid flows to advance ultrasound technology in wastewater treatment.

\section{Ultrasonic Parameters for Acceleration of Fluid Flow and Transport}

In bulk solution near a membrane surface ultrasound can undergo absorption, scattering, reflection and coupling in a similar way as light does. These processes are graphically shown in Scheme 2. Let us consider these parameters in details below.

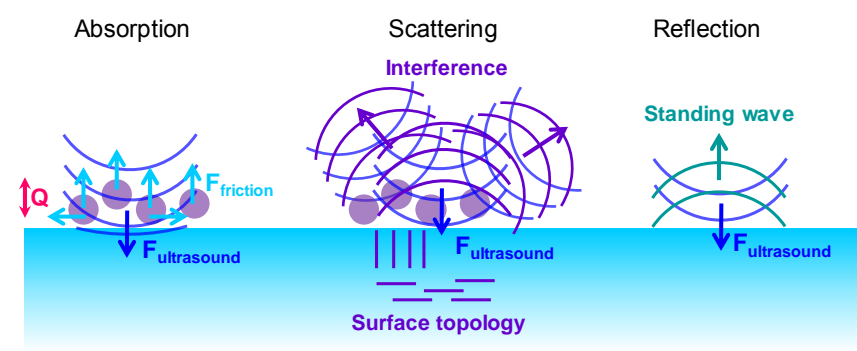

Scheme 2. Schematic illustration of the interaction between ultrasound and a membrane through the processes of absorption, scattering and reflection in liquid solution. In 'absorption' ultrasound causes friction forces $F_{\text {friction }}$ (viscous dissipation) and thermal conduction $Q$. In 'scattering' the incident ultrasound is scattered at different angles followed by the mutual interference. The strength of the interference pattern depends on the surface topology and the incident angle between ultrasound and the filter surface. In 'reflection' ultrasound forms a standing wave between the acoustic source and the filter surface.

\subsection{Absorption}

Absorption of the acoustic energy can occur in form of a fluid flow, which is known as acoustic streaming. ${ }^{[9]}$ Greater rates of this flow can be formed at higher frequency of ultrasound due to stronger absorption. ${ }^{[10]}$ The latter can be higher, if ultrasound impinges on the internal wall of a membrane and forms a larger angle with the wall. ${ }^{[11]}$ To note, an incidence angle close to $\pi / 2$ should be avoided in order to guide ultrasound more efficiently. As the fluid volume changes due to the compression/expansion cycles of the acoustic pressure field, absorption can occur in the fluid itself due to viscous dissipation and thermal conduction. In this way, ultrasound absorption can be characterized by the attenuation coefficient.

If the fluid contains relatively high concentration of particulate solids, attenuation can arise through acoustic impedance. ${ }^{[12]}$ Near a membrane surface frictional attenuation can occur due to dissipation in a boundary layer, ${ }^{[13]}$ where a rotational flow is damped exponentially with the distance from the surface. The vorticity of such a flow decreases with the distance as the square root of the ultrasonic frequency multiplied by the kinematic viscosity (i.e. viscous diffusion rate). ${ }^{[14]}$ Viscous friction and thermal conduction on the surface of the particles are the main parameters of ultrasonic damping due to energy losses. This mechanism becomes more pronounced if particles are of few tens of micron size. In the opposite, smaller particles can be entrained in the acoustic flow field and modify the fluid dynamics.

On the other hand, ultrasound absorption can occur in the fluid itself due to viscosity and thermal conductivity and can be also characterized by an attenuation coefficient. ${ }^{[15]}$ In the presence of particles mechanical action of ultrasound in a fluid can cause attractive forces and form liquid bridges between colloids. ${ }^{[16]}$ Acoustic cavitation can increase the interparticle capillary forces by two or three orders of magnitude per particle mass with dimensions between 0.1 and $0.5 \mathrm{~mm} \cdot{ }^{[17]}$ On the other hand, liquid condensation on the surface of particles can contribute to the development of acoustic streaming. The strength of this phenomenon depends on the relative dimensions of the Stokes boundary layer and the liquid layer. To note, this phenomenon could be a potential subject for future research, as its physical mechanism is not explained.

\subsection{Scattering}

Scattering of ultrasound can occur in all directions due to the heterogeneous composition of the fluid and the membrane surface, leading to overall decrease of its intensity (i.e. energy). The transmitted ultrasonic energy can be decreased due to interference between randomly scattered ultrasonic waves, which are intensified by additional emission from oscillating particles. The topology of the membrane surface and the orientation of ultrasound at the interface can predict the coupling of acoustic waves, i.e. gain or loss in energy. ${ }^{[18]}$ For example, a certain roughness of a surface (e.g. grooved surface with $1 \mu \mathrm{m}$ areas) can lead to an increase in the viscous coupling due to 
larger surface area. In this way, if grooves are parallel to the shear movement, the dependence on the liquid viscosity becomes larger at resonance. If structures are perpendicular to the movement, higher frequency shifts can be observed due to trapped liquid portions (e.g. in water-antifreeze fluid). At a critical angle the amplitude of the reflected wave can be damped due to a viscoelastic loss mechanism in the reflecting material. ${ }^{[19]}$

\subsection{Reflection}

Interference between incident and reflected waves can form an acoustic standing field with a flow velocity around the obstacle in an oscillating boundary layer. This velocity can be increased at higher ultrasonic intensity in fluids with lower viscosity. If the membrane structure contains dissolved gas, highly intense acoustic fields can enhance degassing. Its efficiency depends on the penetration depth of ultrasound in the material. The latter can be predicted from the dissipation of ultrasound energy on the membrane surface, ultrasound scattering and absorption in the viscous gas. In a standing acoustic field Bjerknes forces cause translation of bubbles due to pressure fluctuations between nodes (zero pressure amplitude) and antinodes (maximum pressure amplitude). ${ }^{\left[{ }^{9]}\right.}$ In general, gas bubbles (also lipid vesicles) will be collected at the antinode due to a positive value of a contrast factor, which determines the direction of the acoustic radiation force. In moving to the antinodes, the cavitation bubbles travel in ribbon like structures at velocities approximately an order of magnitude faster than the average velocity of the fluid, coalescing as they collide. ${ }^{[20]}$ This translational motion, micro-streaming, can remove particles from the membrane surface. ${ }^{[21]}$

\subsection{Coupling}

The ultrasonic efficiency determines the intensity of both acoustic streaming and energy at bubble collapse during cavitation. Ultrasound at lower frequency $(20 \mathrm{kHz}-100 \mathrm{kHz})$ leads to higher temperature and pressure gradients and effectively transports or removes particles, which are bound to a surface by molecular or electrostatic forces and capillary attraction. In a fluid bulk the bubble collapse with emanating shock waves, can cause microscopic turbulence. The propagation of shock waves and turbulent flow can strongly increase mass transfer in the surrounding fluid towards the membrane surface. ${ }^{[20]}$ The magnitude and direction of fluid movement occurs with shear or drag forces to the membrane surface. ${ }^{[22]}$ Closer to a surface, bubbles develop micro-jets, which can dislodge particles attached to the surface and break down large aggregates into smaller species. ${ }^{[23]}$ On the other hand, cavitational collapse at higher frequency is energetically less violent, as smaller bubbles produce less heat. ${ }^{[24]}$ If the frequency is in the $\mathrm{MHz}-\mathrm{GHz}$ range, collapse of bubbles cannot occur and rely mainly on acoustic streaming. The parameters of heat transfer at the interface between a fluid and a membrane surface can be determined by means of the Nusselt number. ${ }^{[25]}$ In this case, the ratio of convective (the solid-fluid relative motion) to conductive (stagnant fluid) heat transfer should be considered normal to the boundary.

The quantity of bubbles and the dimension of the cavitation zone can be increased at higher ultrasonic intensity. ${ }^{[26]}$ However, cavitation efficiency increases with increasing power only up to a critical power level. Further increase beyond this critical power level results in a decrease in cavitation activity. ${ }^{[27]}$ This is because the bubble may grow so large on rarefaction, that the time available for collapse is insufficient. Alternatively, at higher power the formation of a cluster of bubbles can be limited to the action of Bjerknes forces. ${ }^{[28]}$ In contrast, fewer bubbles can be formed, if the external pressure is high, as it increases the cavitational threshold. ${ }^{[29]}$ The latter can be lowered at higher temperature (lower viscosity), higher solubility of gas in the fluid and higher diffusion rate of dissolved gases, and increased vapor pressure. Higher ambient temperature will raise the vapor pressure of the fluid and lead to more rapid bubble formation but less violent collapse. At a temperature approaching the boiling point, a very large number of cavitation bubbles can be formed, which can dampen the transmission of ultrasonic energy. ${ }^{[30]}$

\section{Methods for the Acceleration of the Fluid Transport to the Membrane Surface}

\subsection{Mechano-Acoustic Micro-pumps}

There is an increasing demand to develop new ergonomic devices in order to accelerate the fluid transport. Micro-pumps are essential components of such devices, ${ }^{[31]}$ which can be characterized as mechanical or non-mechanical actuation methods. For example, mechanical micro-pumps can be electrostatic, piezoelectric, thermo-pneumatic or magnetic. Among them bulk acoustic wave (BAW) micro-pumps with a mounted piezoelectric element allow precise design of cycleaveraged fluid flows. ${ }^{[32]}$ The flow rates can be predicted by computing the time-averaged velocity fields. In a typical BAW micro-pump construction (Figure 1) fluid flow can be modelled by using mechanical equations of motion for the solids, the electromagnetic field equations for the actuator and mass/momentum conservation equations for the fluid.

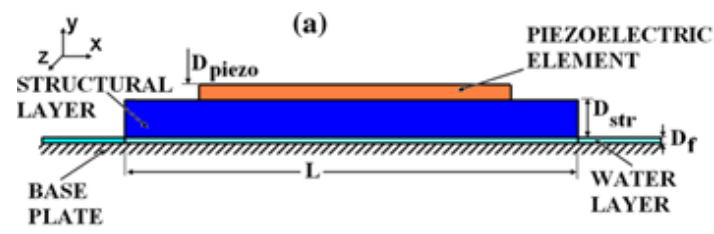

(b)

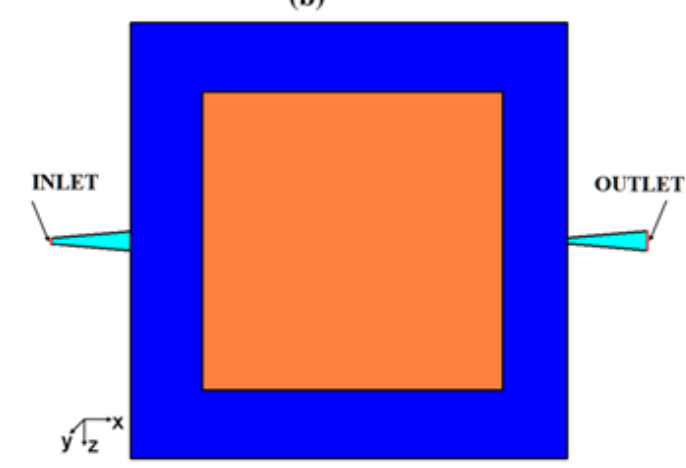


Figure 1. Piezoelectric BAW micropump geometry in a) front view and b) top view. Flows can be generated in the piezoelectric BAW micropump and can be modeled using mechanical equations of motion for the solids, the electromagnetic field equations for the actuator and mass/momentum conservation equations for the fluid. The piezoelectric BAW micro-pump device configuration with the double-chamber cylindrical pump geometry has a thin-film bulk acoustic resonator (FBAR) structure. The BAW micro-pump consists of trapezoidal prism inlet/outlet elements and the pump chamber (bottom layer); one thin structural layer (middle layer) and one piezoelectric element (top layer) as the actuator. Pumps considered in the present work are made of Pyrex 7740 borosilicate glass structural layer and PZT-5A piezoelectric element. The length $(\mathrm{L})$ and the width of the pump chamber are both equal to $6.0 \mathrm{~mm}$. Length and width of the piezoelectric element are 3.8 $\mathrm{mm}$. The depths of the (un-deformed) pump chamber and the trapezoidal prism inlet/outlet are $80.0 \mu \mathrm{m}$. At $\mathrm{x}=7.1 \mathrm{~mm}$, the outlet of the micro-pump is positioned at the far right. Thickness of the structural layer, Pyrex 7740 borosilicate glass is $500.0 \mu \mathrm{m}$ and the thickness of the piezoelectric element, PZT-5A is $200.0 \mu \mathrm{m}$. A water layer is formed under the Pyrex glass $(0 \mu \mathrm{m}<\mathrm{y}$ $<80.0 \mu \mathrm{m})$ followed by the Pyrex glass $(80.0 \mu \mathrm{m}<\mathrm{y}<580.0 \mu \mathrm{m})$ and finally the PZT-5A element $(580.0 \mu \mathrm{m}<\mathrm{y}<780.0 \mu \mathrm{m}$. The top of the micro-pump ( $=780.0 \mu \mathrm{m})$ is free to deform. Reprinted with permission from the reference $\mathrm{E}$. Sayar, B. Farouk, Microfluid Nanofluid (2015) 18, 433-445.

In the BAW piezoelectric micro-pump the double-chamber cylindrical geometry includes a thin-film bulk acoustic resonator structure. Working fluid (water) entirely fills the fluid domains in the inlet, pump chamber and outlet elements. The fluid properties of water can be treated as compressible and Newtonian with its velocity and pressure field in the frame of three-dimensional continuity and momentum equations. ${ }^{[3]}$ For isentropic waves, the pressure is linearly related to the density through the speed of sound in the fluid. This isentropic assumption is valid for ultrasound with frequency higher than $200 \mathrm{~Hz}$, where the isentropic equation of state can be applied. ${ }^{[34]}$

The coupling of the mechanical, electrical and fluid fields occurs in structural near-surface layer through interaction with the liquid at its bottom surface and creates displacements in normal and tangential directions. The variation of design parameters such as applied electric potential, actuation frequency and geometric variables (the thickness of the glass layer and piezoelectric elements, inlet/outlet lengths and taper angles) can significantly accelerate the mass transport, which is otherwise diffusion limited. For flow with acoustic excitation the energy transmitted by the oscillating wall is attenuated not only by the inertia and viscosity of the fluid, but also by the density variations (accounted for in the present study) in the fluid. Higher transport rates occur at higher actuation frequency, where the elasticity of the piezoelectric element and structural material is less dominant. Net fluid flow increases with increasing excitation voltage up to a certain value, when the instantaneous pressure is higher than the vapor pressure at a given temperature. Both actuation frequency and excitation voltage of the micro-pump are limited by acoustic cavitation (bubble formation, growth and collapse).

\subsection{Thermoacoustic Wave Emission}

The mass transport can be accelerated by producing acoustic waves (expansion/contraction cycles) near a solid wal in a compressible medium with a temperature gradient. ${ }^{[35]}$ Periodic heating/cooling phases in the localized region propagate at a local speed of sound, and their amplitudes are limited to thermal and viscous diffusion. The acoustic pressure in this field can be predicted for any solid material by using a thermal piston model for ultrasonic radiation from a thermoacoustic transducer. ${ }^{[36]}$ The strength of the thermoacoustic emission is limited to heat conduction and capacity of a thin conductive membrane and the surrounding medium. The choice of suitable materials can be extended to porous silicon and graphene, which significantly enhance the Joule heating process through electron transfer during absorption of electric energy. In a typical thermoacoustic emission system (Figure 2) temperature and pressure perturbations can be determined at the interface of the thin conductive membrane.

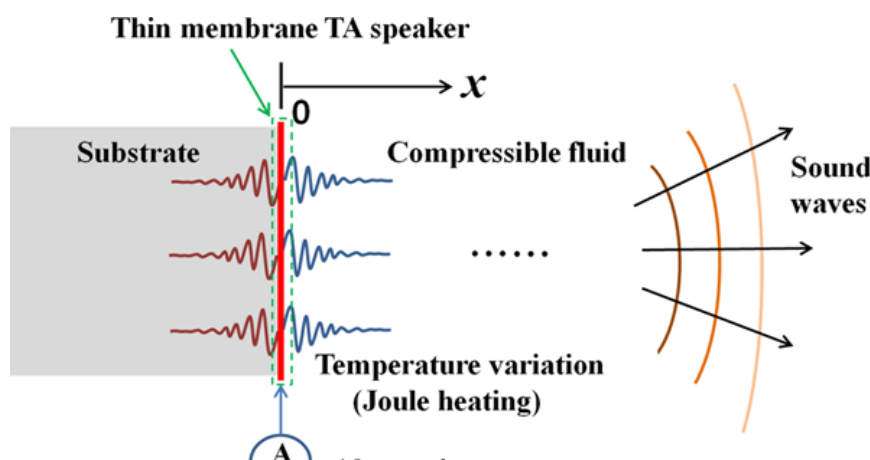

Figure 2. Schematic illustration of the thermoacoustic wave emission representing the temperature change in the thin membrane by Joule heating The origin of coordinates is placed on the interface between the sample (graphene) and gas. The gas in the compressible medium is assumed to be ideal. The governing equations for the linear thermoacoustic problem in isotropic materials can be given by the heat conduction and wave equations describing the coupling of pressure and temperature fields. Isentropic sound speed is used for the pressure in the far-field by assuming that temperature variation only exists near the wall. By assuming that the magnitude of the sinusoidal pressure fluctuation in the thermal boundary layer is proportional to the amount of heat emanating from the solid, this layer of air is regarded as an acoustic piston generating sinusoidal pressure fluctuations propagating into the far field. Reprinted with permission from the reference J. Bin, W. S. Oates K. Taira, J Appl. Phys. 117, 064506 (2015).

At the graphene interface heat transport, temperature and pressure waves in the surrounding media are in contact. In the latter unknown heat transport parameters can be modelled by using Bayesian analysis with the Markov Chain Monte Carlo method $^{[37]}$ in the range of emission of acoustic waves from $1 \mathrm{kHz}$ to $30 \mathrm{kHz}$. In contrast to the acoustic wavelength, the thermal wavelength is smaller and decays exponentially with the distance from the wall. Bayesian statistics suggests that the heat flux and the heat capacity can dominate thermoacoustics, while heat convection can be negligible in ambient condition. The acoustic response with respect to the frequency shows different characteristics when assuming Dirichlet (temperature) or Neumann (heat flux) boundary conditions. Overall, this method can be useful in acceleration of mass transport by heating with sound, which can be produced by thermal cycling of a thin conductive membrane at high frequencies. 


\subsection{Acoustophoretic Transport near Viscoelastic Surface}

Solids and liquids at a smaller scale can be effectively transported by acoustic forces in a new concept, which is based on acoustophoresis of a deformable reflector. ${ }^{[38]}$ In this method, a thin overlaid membrane can act as a morphing reflector with low reaction forces and relaxation time of a liquid, but requires higher surface tension. A reflector surface enhances the acoustic field through a mobile deformation and enables the continuous motion of levitated mass volumes. The mass transfer is contactless and can effectively transport any dense material (e.g. steel sphere or alloys) independent of its physical properties (e.g. electrostatic, electromagnetic or optical). As an advantage this approach eliminates possible contamination through a contactless procedure and pretreatment steps. Although this method is successfully demonstrated in air, it can be extended to a liquid due to contribution of buoyancy forces. The mutual interaction between the reflector and emitter depends on their geometry and can enhance the acoustic field by several orders of magnitude. ${ }^{[39]}$ Acoustic power, as another parameter, can increase the radiation pressure in order to deform the surface of liquids or soft materials. ${ }^{[40]}$ The deformation changes in turn the acoustic resonance frequency of the acoustic levitator. This leads to modification of the acoustic field: the steady-state nature of the radiation pressure couples with the reaction forces of the reflecting material. A stronger focusing effect of the acoustophoresis can be obtained by reducing oscillations at the potential nodes through the curvature geometry of a reflector and a medium with a higher damping effect. As an example, a plastic wrap membrane can be stretched on the surface of a liquid with the properties for morphing through acoustic radiation pressure (Figure 3 ).

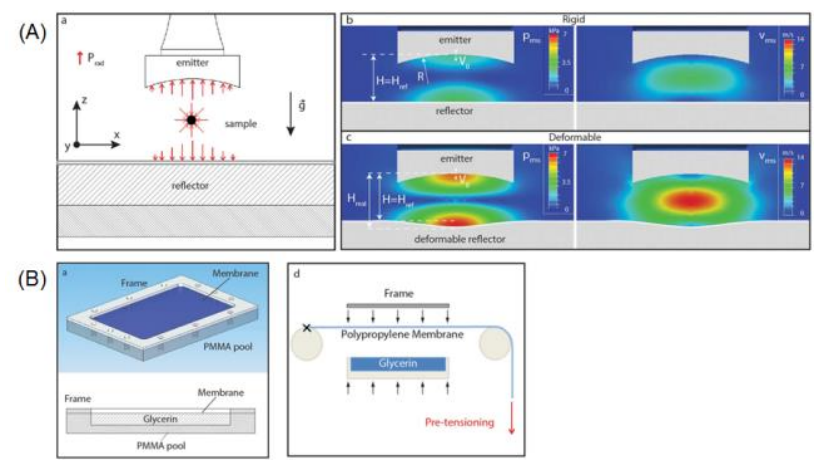

Figure 3. Figure Caption. A) In (a) a line-focused acoustic levitator, front view The radiation pressure acts on the emitter, reflector and levitated sample. To achieve higher force, the emitter is placed at a resonance distance $\mathrm{H} \approx N / 2$, from the reflector where $\lambda$ is the acoustic wavelength. (b) Rigid configuration of a line-focused levitator, front view, along with the acoustic $p_{r m s}$ and $v_{r m s}$ (root mean square acoustic pressure and velocity) distributions. A standing wave can be established with a single vertical acoustic node, where the sample is placed. (c) A deformable reflector morphs under radiation pressure enhances the acoustic field. If soft-enough the reflector can deform under the effect of the radiation pressure. For a constant emitter oscillation frequency $f$ and velocity amplitude $V_{0}$, both acoustic pressure and particle velocity depend on the levitator geometry. The velocity $V_{0}$ is the same in the rigid case, and in this specific configuration the acoustic pressure and velocity increases by a factor of 2 by the sole morphing of the soft reflector. By varying the height $\mathrm{H}$ in the proximity of $\lambda / 2$, the resonant height $H_{r}$ of the levitator can be found, which corresponds to the strongest acoustic field. $H_{r}$ also depends on many other geometrical parameters with the most influencing factor being the curvature of the reflector. B) On the left, in (a) isometric and lateral views of the reflector structure. On the right, in d) the structure assembly, pre-tensioning allows the calibration of the final stiffness. Reprinted with permission from the reference D. Foresti, G. Sambatakakis, S. Bottan, D. Poulikakos, Scientific Reports 3, Article number: 3176 (2013).

The surface deformation can assume a concave shape, which moves along the transported volume and forms a spatially and temporally optimized deformation along its propagation. The acoustic force depends on the morphed geometry, and vice versa. Among the suitable materials is polypropylene membrane on a glycerin surface, as it exhibits a linear dependence from a Young's modulus in a wide range of radiation pressure. In contrast, polydimethylsiloxane (PDMS) can be insufficiently cross-linked and act as a jelly-like reflector with adhesive properties. Overall, hydrogels exhibit an inherent dependence of mechanical properties on their hydration state, temperature and stress, which can alter the material response with time. Another limitation is the shorter time scale of the acoustophoretic transport ( $<1 \mathrm{~s}$ ) and longer relaxation time (seconds). As another restriction, at higher acoustic power the direct contact with the acoustic field can be prohibited if only a liquid is used. If acoustic pressure is focused on a point at higher power, it can destroy the continuity of the liquid surface and trap macrobubbles. For this reason the most suitable reflector composition could be a hybrid liquid/membrane system, which combines the advantages of both components.

\subsection{High Frequency Ultrasound and Magnetic Stirring}

Increasing the transport properties of ion-exchange membranes has considerable importance for treatment of industrial and biological effluents, desalting brackish water, changing the concentration of brine from seawater or producing table salt. However, these properties can be limited to a diffusion boundary layer especially at low solution concentration, which increases the total resistance. One of the methods to increase the mass transport is the combination of ultrasound at higher frequency $(\mathrm{MHz})$ with magnetic stirring. ${ }^{[11]}$ Acoustic cavitation at a frequency of ultrasound higher than $600 \mathrm{kHz}$ forms bubbles of smaller radius and cause them to oscillate without an implosive collapse (i.e. stable cavitation). In this way bubbles create micro agitation of the surrounding medium and cause micro-streaming without shock wave or jet formation. At the same time high frequency ultrasound is absorbed stronger favoring acoustic streaming. The latter can thus be increased at higher frequency and intensity of ultrasound. In this way, mass transfer can be accelerated due to an increased fluid movement by propagation of micro-streams. One of the schematic diagrams of a test cell with piezoelectric transducer and magnetic bar with a stirrer is shown in Figure 4

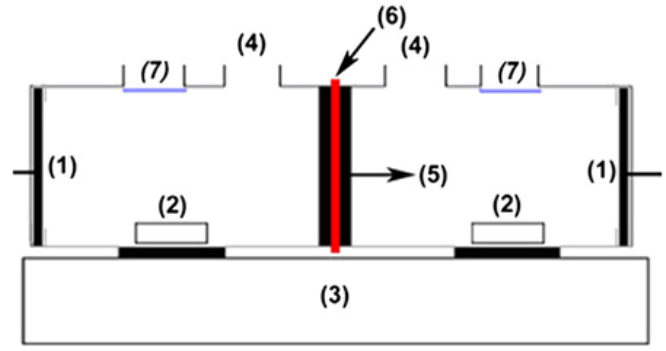


Figure 4. Schematic diagram of a test cell: (1) Pt electrode, (2) magnetic bar, (3) stirrer, (4) orifice, (5) Rubber ring, (6) membrane, (7) piezoelectric transducer. The electrodialysis ion-exchange membrane module has the radius of $2.1 \mathrm{~cm}$ and the length of $10 \mathrm{~cm}$ for each compartment. Two piezoelectric transducers can be employed on the positions with the frequency $\mathrm{f}=1.7 \mathrm{MHz}$, velocity $\mathrm{c}=1480 \mathrm{~m} / \mathrm{s}$ and acoustic pressure $\mathrm{PA}=2.8 \times 10^{5} \mathrm{~Pa}$ Magnetic stirrers can be considered as cylinders with radius of $0.5 \mathrm{~cm}$ and height of $1 \mathrm{~cm}$. The magnetic stirrer rotation speed can be maintained at a constant value of $1250 \mathrm{rpm}$. Reprinted with permission from the reference $F$. Parvizian, M. Rahimi, S. M. Hosseini, S. S. Madaeni, A. A. Alsairafi, Desalination 286 (2012) 155-165.

The modelling of the fluid transport at the boundary of the membrane shows circular patterns around the magnet's rotation axis with the formation of downward and upward loops in the regions of the acoustic pressure field (Figure 5). The velocity of fluid transport towards the membrane surface increases, if the acoustic field is combined with magnetic stirring, but not within the membrane structure. In the vicinity of a membrane stronger acceleration can be caused by ultrasound alone. Enhancement of fluid flow by ultrasound is about two times higher than by magnetic stirring (Figure 5)

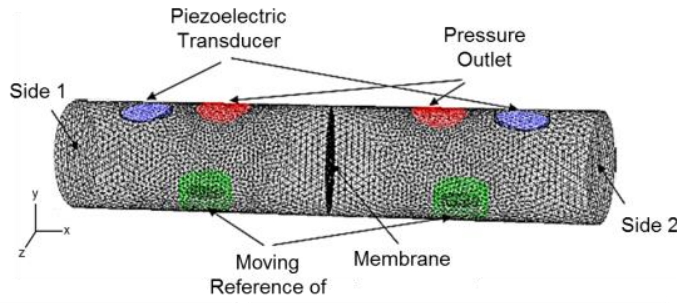

Frame

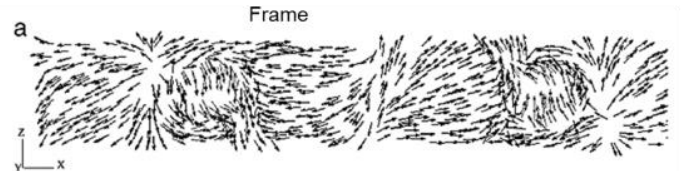

(

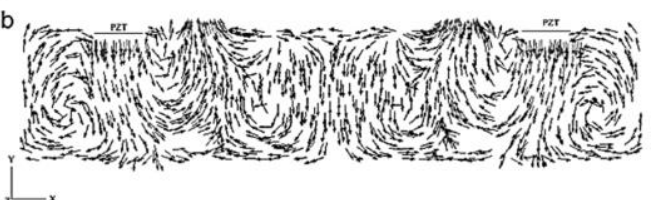

(a) Before imposing the

(b) After imposing the ultrasonic beam ultrasonic beam

(c) After imposing the
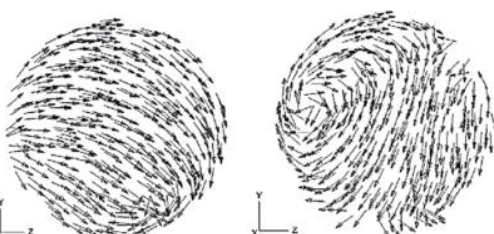
stationary magnetic stirrer

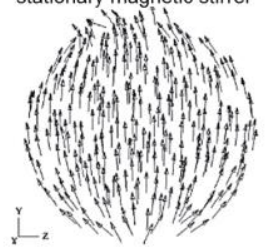

Figure 5. Figure Caption. The 3-D computational domain and boundary conditions of the membrane system with decorated piezoelectric transducers (first top row). In the second middle row the velocity vector plots after the exposure of ultrasound at a) horizontal and b) vertical slices. In the lowe bottom row velocity vector graphs near the membrane surface a) before and b) after the exposure of ultrasound and c) after ultrasonic treatment with a magnetic stirrer. In the modeling the operational conditions are $\mathrm{T}=298 \mathrm{~K}$ $P=101.3 \mathrm{kPa}$ and $\mathrm{f}=1.7 \mathrm{MHz}$. Direction of velocity vectors indicates circular pattern around the magnetic rotation axis. After activation of PZTs they are significantly affected by ultrasound in the liquid followed by the formation of downward and upward loops. After imposing the ultrasonic beam the maximum velocity in the vicinity of the membrane surface can increase about 1.5 and 2.6 times with and without magnetic stirring. It is remarkable that using ultrasound alone can generate higher fluid velocity than using both ultrasound and magnetic stirrer. This is because the streams created by ultrasonic are influenced by streams created by the magnetic stirrer. Reprinted with permission from the reference F. Parvizian, M. Rahimi, S. M. Hosseini, S. S. Madaeni, A. A. Alsairafi, Desalination 286 (2012) 155-165.

Ultrasound decreases the membrane electrical resistance due to an increased mixing of an electrolyte solution and higher mass transfer rates. On the other hand, acoustic streaming reduces the thickness of the Nernst diffusion layer. Heterogeneities at the membrane surface can provide additional mixing in the diffusion boundary layer due to hydrodynamic instability. ${ }^{[42]}$ At higher concentration of an electrolyte ultrasound exhibits stronger effects on the membrane potential, transport number and permselectivity due to higher concentration polarization. At lower concentration of an electrolyte ultrasound propagation becomes more pronounced, as it creates higher turbulent flows towards the membrane surface. These can prevent co-ion percolation throughout the membrane and improve the membrane selectivity. ${ }^{[41]}$

\subsection{Acoustic Entrapment of Bubbles in Micro-Fluidic} Environment

There is a need to increase the fluid flow in the laminar pattern due to the low Reynolds number in the restricted environment in a microfluidic chip. Among various microfluidic systems, which employ chaotic advection, ${ }^{[43]}$ hydrodynamic focusing, ${ }^{[4]}$ and mixing by electrokinetic, ${ }^{[45]}$ thermal ${ }^{[46]}$ or optical ${ }^{[47]}$ means, acoustic-based systems are of great interest due to their non-invasive nature. Other advantages of the latter include simple, inexpensive fabrication procedures and facile instrumentation. The fluid laminar flow pattern can be disturbed through pressure fluctuations created by ultrasound with a further increase due to the oscillation by gaseous bubbles. These bubbles can focus acoustic energy due to the coupling with acoustic waves ${ }^{[48]}$ and significantly enhance the mass transport. In a typical construction device microbubbles can be trapped in the sidewall grooves of a chamber, which are decorated by piezo transducer disks at the backside to generate acoustic waves (Figure 6). 


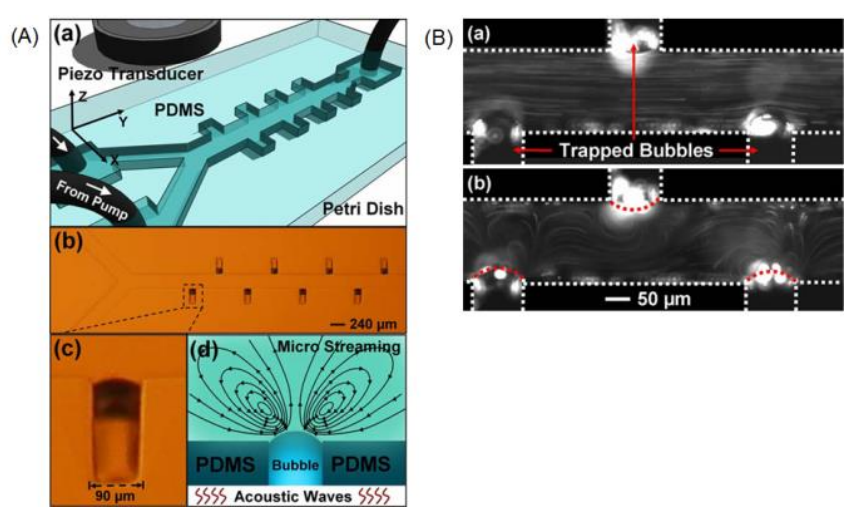

Figure 6. A) On the left, a single-layer Y-shaped polydimethylsiloxane (PDMS microchannel with two inlets and one outlet. The channel features rectangular grooves on the sidewalls designed with alternate spacing. As liquid was injected into the inlets, bubbles were trapped inside the sidewall grooves due to surface tension $(b, c)$. The trapped air bubbles are externally excited by acoustic energy from a piezo transducer, causing the membranes (air/liquid interfaces) to oscillate. As a result frictional force was developed between the boundary of the bubble and the surrounding medium. This oscillation generates pressure and velocity fluctuations in the surrounding liquid, resulting in a phenomenon known as micro-streaming. The streaming pattern (d) is shown by a pair of counter-rotating vortices. The streaming drastically perturbs the bulk fluid flow, breaks the laminar fluid interface and enhances the mass transport between the two fluids, thus making fast and homogenous mixing possible. B) On the right, the flow pattern of fluorescent bead solution with microbubbles that induce strong acoustic streaming. In a) the flow pattern of fluorescent bead solution prior to exciting the microbubbles, and b) once activated, bubbles induce strong acoustic streaming phenomena. The streaming effects drastically perturb bulk laminar flow and enhance the mass transport across the centerline of the channel thus making fast and homogeneous mixing possible. Reprinted with permission from the reference D. Ahmed, X. Mao, B. K. Juluri, T. J. Huang, Microfluid Nanofluid (2009) 7:727-731.

Streaming occurs due to bubble oscillation, which is induced by ultrasound from a vibrating transducer. Such an oscillation generates fluctuations of pressure and velocity in the surrounding liquid (micro-streaming). It also develops a frictional force at the boundary between bubble and medium. ${ }^{\left[{ }^{[3}\right.}$ Streaming perturbs the bulk fluid flow, breaks the laminar fluid interface and enhances the mass transport. To note, the effective mass transport can be observed across the width of the channel within shorter time (< $120 \mathrm{~ms}$ ). This method can be used in a wide variety of "lab-on-chip" applications that require fast and homogeneous mass transfer due to its simple design, stronger performance and faster speed.

\subsection{Structural Acoustic Coupling}

To reduce noise, which is formed by structural acoustic interaction, many efforts have been devoted to understand the physical mechanism connecting structural vibration and fluid pressure distribution over the structure. ${ }^{[49]}$ The latter can be modelled through the design of an efficient radiator in order to predict the pressure distribution and radiation pattern of a structural-acoustic coupling system (Figure 7). Such a radiator system can be composed of two spaces, which are separated by a wall with transporting acoustic characteristics (Figure 7A). The spaces can be categorized as bounded finite space and unbounded infinite space. The wall involves two plates and an opening, and a separated space, which is highly reverberant and the other one, which is unbounded without any reflection. The radiator structure can represent a "volume interaction" element, and the opening can be considered as a "pressure interaction" element and can be mathematically modelled (Figure 7B). The velocity distribution of the accelerated fluid flow has its maximum value at the cutting edge and develops singular-like features near the edges (Figure 7C). This method allows prediction of the energy distribution (pressure) and energy flow (active intensity) of two spaces in the coupling system's function. Three types of radiation patterns, which are steered, focused, and omnidirected can be estimated. The steered beam can be applied to achieve noise control, and the focused beam can be used for designing an efficient radiator or directivity pattern.
(A)
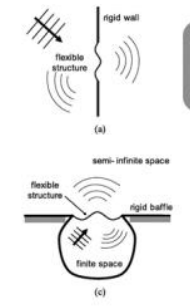

(C)

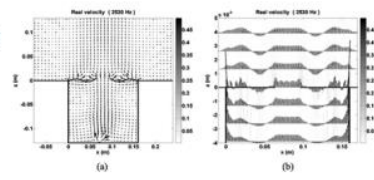

(B)
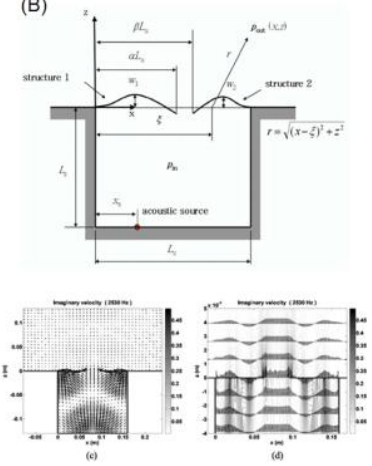

Figure 7. A) Typical examples of coupling systems: a) unbounded spaces separated by the wall; b) two bounded spaces separated by the wall; c) bounded and unbounded spaces separated by the wall; and d) bounded and unbounded spaces separated by two walls with an opening. B) The mathematical model of a coupled cavity, which can be used in the study. The cavity is rectangular and its size is $L_{x}$ by $L_{z}$. The plates have length $L_{x}$ and $1-L_{x}$, respectively. The source is located at the bottom of the cavity ( $z=-L_{z}$ $x=x s)$. C) The velocity distribution of two acoustic spaces at the fifth acoustic dominated mode $2530 \mathrm{~Hz}$ about the coupled system. In a) real part of the whole cavity and external space; b) real part near the opening; c) imaginary part of the whole cavity and external space; d) imaginary part near the opening. The velocity at the cutting edge is maximum and it has singular-like features near the cutting edge. Reprinted with permission from the reference $H$. S. Seo,Y. H. Kim, J. Acoust. Soc. Am. 118, 1, 2005

\subsection{Electroconvection}

In electrodialysis a non-stationary current (pulsed or alternating) can increase the mass transfer and mitigate the membrane fouling. Higher fluxes occur, if the diffusion boundary layer is reduced by current-induced convection (Figure 8) (i.e. electroconvection). ${ }^{[50]}$ In electroconvection an electric force is directed normally to the membrane and creates an excessive pressure within the surface charge region (SCR) near the interface. This excessive pressure displaces the liquid from the SCR in tangential direction towards zones with lower pressure (= lower space charge). Upon movement the displaced fluid meets inertial resistance of non-slipped liquid layers. The latter change the direction of a moving fluid towards the bulk solution and create a pair of vortices with opposite rotation (Figure 8A). This type of convection is a more likely mechanism of intensive electro-convective fluid transport at overlimiting currents. Vortices can be formed if the SCR is non-uniformly distributed 
along the interface, which can take place near a heterogeneous membrane and a curved surface. The speed of electroconvective motion may depend not only on the space charge and applied electric force values, but also on the Stokes radius of counterions forming the surface charge region. To note, the Stokes radius of an ion is the radius of a hard sphere, close to that of the hydrated ion, which exhibits the same hydrodynamic property as the ion. Higher volume of a liquid can be involved in the motion at a larger radius. To note, $\mathrm{H}^{+}$and $\mathrm{OH}^{-}$ions have a rather small Stokes' radius due to the Grotthuis transport mechanism of these ions. They carry the charge by "tunnelling" from one water molecule to another without bringing liquid volume into motion. In this way, water splitting can suppress the electro-convective mass transport. Non-equilibrium electrokinetic vortices can appear due to the non-equilibrium electroosmotic flow near a nanochannel (Figure 8B). The enhancement of the fluid transport can be monitored by simultaneous registration of current-voltage curves at overlimiting current. ${ }^{[51]}$
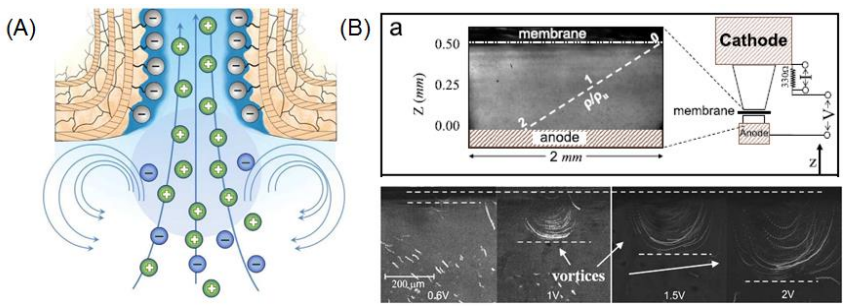

Figure 8. Figure Caption. (A) scheme showing the emergence of electroconvection at the entrance of a membrane nanopore with charged walls. (B) In the top row, a schematic view of the experimental system consisting of a horizontal transparent PMMA cell, which is sealed by a massive, polished copper anode at the bottom and a cation-exchange membrane at the top. A massive copper cathode is sealed above the membrane. The voltage bias, $V$, is applied directly to the cell in series with a $330 \Omega$ resistor, allowing the detection of the current, $I$. The electrolyte solution is seeded with $1 \mu \mathrm{m}$ polystyrene tracer particles. The dashed line illustrates the concentration polarization profile at the limiting current. In the bottom row, time-lapse snapshots of the experimental cell seeded with tracer particles showing 'quasisteady-state' streamlines. The membrane is situated at the top boundary of each image, the applied voltage (Volts) is indicated. Reprinted with permission from the references V. V. Nikonenko, N. D. Pismenskaya, E. I. Belova, P. Sistat, P. Huguet, G. Pourcelly, C. Larchet, Advances in Colloid and Interface Science 160 (2010) 101-123 and Rubinstein SM, Manukyan G, Staicu A Rubinstein I, Zaltzman B, Lammertink RGH, F. Mugele, and M. Wessling Phys Rev Lett 2008; 101, 236101.

Overall, when a pulse of constant voltage is applied to an equilibrium membrane/solution system, the electromigration contribution to counterion transfer is increased, although the concentration gradients are initially absent. Then the concentration gradients develop producing a decrease in concentration at one side of the membrane and an increase at the other. If the pulse duration is sufficiently long, the system, after a transition time, achieves a steady state where the difference of electromigration fluxes in membrane and solution is compensated by the diffusion fluxes. When the frequency is sufficiently high, the concentration profile remains steady everywhere except in a thin region near the membrane surface, where the concentration follows the current oscillations.

The advantage, in comparison to the steady state, is that only a small part of the concentration profile attains very low concentrations. This seems sufficient to produce a space charge and to initiate electroconvection. At the same time, the electrolyte concentration is not so low, that it assures a rather good conductance. The gain in applying pulsed currents may be also due to inertial properties of liquid movement. Hence, current-induced convection can exist not only during the application of the voltage pulse, but also in the period of pause. This residual convection could partially restore the electrolyte concentration near the membrane. If the duration of the voltage pulse is high, the fluid vortex may partially destroy the space charge region by introducing a portion of "fresh" solution from the bulk. If the duration of the pulse is short, but the pulse is strong, it can produce a force and bring the liquid into motion.

As we have shown above, only few effective methods exists now, which employ ultrasound or acoustic cavitation to accelerate the fluid flow to the membrane filter. Most of them can be controlled by ultrasonic frequency and intensity and can determine micro- and acoustic streaming. The latter is the main driving source for mass transport towards the filter. At a closer distance to the filter ultrasonic streaming exhibit forces at the boundary surface layer and can remove contaminants. In this way, smart design of a filter flow by ultrasound can not only clean the filter surface, but prevent the antifouling. Let us examine the physico-chemical principles and approaches of ultrasonic antifouling and its enhancement in filtration.

\section{Ultrasonic Antifouling of Membrane Filters}

\subsection{Cleaning Effects of Ultrasound}

Effective membrane operation depends on prediction and exclusion of the predominant fouling mechanisms, which fall into three categories. They are i) adsorption of fouling material on the membrane surface or in the pore walls; ii) the build-up of a cake or a gel-like layer on the membrane surface and iii) blocking of membrane pores. ${ }^{[52]}$ For continuous membrane operation both physical and chemical cleaning methods can be used. Physical cleaning includes mechanical action on a membrane followed by backwashing cycles. The task of chemical cleaning is to completely recover the membrane flux by using caustic or oxidizing chemicals. ${ }^{[53]}$ However, chemical methods produce toxic or contaminated wastewater, and most physical procedures are energetically unfavorable.

In general, the deposition of particles, colloids and macromolecules on a membrane surface leads to an external fouling with formation of a 'fouling layer' on the membrane surface. External fouling consists of a gel layer due to the precipitation of particulates and a cake layer due to the accumulation of retained solids on the membrane.$^{[54]}$ Both types of fouling layers require physical and/or chemical means of cleaning. ${ }^{[55]}$ In physical cleaning loosely attached materials could be removed from a membrane surface ('reversible fouling'), while more tenacious compounds could be cleaned by chemical means ('irreversible' fouling). ${ }^{[56]}$ Physical cleaning includes mechanical action on a membrane followed by backwashing cycles. The task of chemical cleaning is to completely recover 
the membrane flux by using caustic or oxidizing chemicals. ${ }^{[33]}$ However, chemical methods produce toxic or contaminated wastewater and most physical procedures are energetically unfavorable. The adsorption and deposition of solutes and fine particles within the internal structure of membranes, e.g. to the pore-walls, resulting in pore narrowing or blocking, causes internal fouling. Harsh mechanical cleaning cannot completely remove fouling as both feed and permeate side of the membrane undergoes biofouling. ${ }^{[57,58]}$

Among other methods, ultrasound can combine both types of cleaning. As a result it can overcome secondary chemical contamination, significantly reduce the quantity of chemical reagents and accelerate the membrane filtering. Overall faster mass transport towards and near a membrane surface can be determined by acoustic cavitation and streaming. ${ }^{[59]}$ Flux enhancement due to turbulence and antifouling due to acoustic streaming are the consequences of bubble collapse, which becomes more asymmetric closer to the membrane surface. Acoustic cavitation can cause agglomeration of fine particles and significantly reduce pore blockage and cake compaction. ${ }^{[60]}$ It can be also used for separation of molecular aggregates by disrupting the intermolecular forces. ${ }^{[59]}$ Scrubbing of a surface, removal of a fouling layer and reduction of a concentration polarization can be attributed by the asymmetrical collapse of cavitation bubbles.

\subsection{Ultrasonic Mechanisms of Antifouling}

Overall, fouling depends on the physical and chemical parameters of the filtrate solution (e.g. composition, temperature, $\mathrm{pH}$, viscosity, ionic strength), surface characteristics of the membrane (hydrophobicity and the nature of a surface charge) and hydrodynamics (cross flow velocity, turbulence and transmembrane pressure). ${ }^{[61]}$ How to control the fouling? One of the ways could be to pretreat the feed solution in order to modify or reduce the foulant material. Another approach could be to modify the membrane surface so that the affinity of foulants for the membrane surface can be reduced. The rates of both fouling attachment and shear-induced removal can be increased by optimization of the cross flow velocity and transmembrane pressure. $^{[61]}$ The latter can be significantly advanced by the effects of acoustic cavitation.

Ultrasonic removal of contaminated surface layers (external fouling) involves two mechanisms, which are based on the interaction of bubbles with a cake or gel-like film. ${ }^{[62]}$ The first one is the destruction associated with the annihilation of cavitation bubbles. In this case the disappearance of the bubbles will be immediately followed by the tearing of a large piece of the contamination layer. The second mechanism is observed, when small bubbles penetrate the pores and gaps between the contamination layer and the surface. Their vibration peels and tears off pieces of the contamination layer. ${ }^{[3]}$ However, both mechanisms cannot explain ultrasonic antifouling of a contaminant layer, which consists of individual particles rather than a film.

Another mechanism of ultrasonic antifouling relies on an increased hydrodynamic flow near a membrane surface, i.e. acoustic streaming, which appears in an oscillating viscous fluid. ${ }^{[64]}$ Acoustic streaming has a rotational character with the velocity increasing at higher ultrasonic intensity. ${ }^{[65]}$ Streaming can occur either inside (Schlichting) or outside of the acoustic boundary layer, where it causes the removal of small particles from surfaces. This type of streaming is characterized by vortices of a scale being much smaller than the wavelength. The dimension of these vortices is proportional to the acoustic boundary layer thickness and develops large velocity gradients during interaction with a solid boundary. As a result steady viscous stresses can be exerted on the boundaries with this type of circulation and significantly contribute to the removal of surface layers. ${ }^{[6]]}$ The streaming velocity can be increased at higher frequency and power and lower kinematic viscosity. ${ }^{[67]}$ At larger distance from the source this velocity decays due to attenuation. In spite of extensive theoretical work, little attention has been paid to the particle removal process by this type of acoustic streaming. ${ }^{[68]}$

Acoustic cavitation can form a round deep impression on the surface of the cake layer on the ceramic membrane, which is fouled with polystyrene sulfate particles (Figure 9A and B). This indentation does not disturb the latex particles, which are directly adjacent to the formed defects. The shape and size of these defects are similar to a damage, which is caused by micro-jets on metal surfaces (51). The average diameter of these cavities is about $18 \mu \mathrm{m}$ and does not depend on the selected frequency (e.g. $620 \mathrm{kHz}$ and $1062 \mathrm{kHz}$ ) and power density (e.g. 0.21 $\mathrm{W} / \mathrm{cm}^{2}$ and $0.12 \mathrm{~W} / \mathrm{cm}^{2}$ ) of ultrasound. The second type of deformation appears as a circular patch of removal with an average diameter of about $2.3 \mathrm{~mm}$ (Figure 9C and D). As acoustic cavitation starts, the patch appears rapidly with extent increasing with time, until it covers the entire surface. The latter acquires a void of latex particle fouling on the membrane surface and can be considered as the main source of particle removal. The edges of these circular patches of removed particles develop channels with about $1 \mathrm{~mm}$ length and several $\mu \mathrm{m}$ widths. These channels could be attributed to micro-streaming, which is caused by localized high fluid velocities near the fouled interface. Alternatively, these channels could be formed due to bubbles travelling along the membrane surface moving particles towards an antinode. ${ }^{[69]}$ Bubbles could move in channel-like paths, merge with one another and end up at an antinode. Coupling with microscopic turbulence could increase the erosion of the fouling layer. 

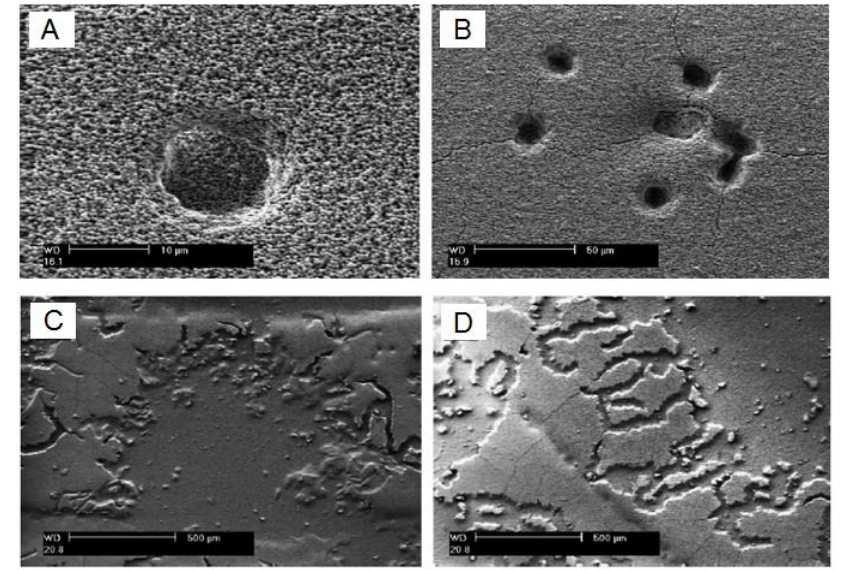

Figure 9. Figure Caption. SEM images of an anodisc ${ }^{T M} y$-alumina $\left(y-\mathrm{Al}_{2} \mathrm{O}_{3}\right)$ ceramic membrane $(25 \mathrm{~mm}$ diameter and $0.2 \mu \mathrm{m}$ pore size), which is fouled with sulfate polystyrene latex particles $(0.53 \mu \mathrm{m}$ diameter) after $5 \mathrm{~s}$ of ultrasonic treatment at A) $1062 \mathrm{kHz}\left(0.21 \mathrm{~W} / \mathrm{cm}^{2}\right)$ and B) $620 \mathrm{kHz}(0.12$ $\left.\mathrm{W} / \mathrm{cm}^{2}\right)$. Acoustic cavitation forms a round deep impression on the surface of the cake layer. This indentation does not disturb the latex particles, which are directly adjacent to the formed defects. The shape and size of these defects are similar to a damage, which is caused by micro-jets on metal surfaces. SEM images of circular patches of cake layer removal and channel-like formations along the edges after $5 \mathrm{~s}$ of ultrasonic treatment at C) $620 \mathrm{kHz}$ $\left(0.42 \mathrm{~W} / \mathrm{cm}^{2}\right)$ and D) $205 \mathrm{kHz}\left(0.21 \mathrm{~W} / \mathrm{cm}^{2}\right)$. Circular patches with channels on the perimeter can be attributed to micro-streaming/micro-streamers. Reprinted with permission from the reference M. O. Lamminen, $\mathrm{H}$. W. Walker, L. K Weavers, Journal of Membrane Science 237 (2004) 213-223.

In another type of streaming (acoustic micro-streaming), which occurs near bubbles in the irradiated liquid, these bubbles scatter ultrasound and locally generate swift currents. ${ }^{[0]}$ These currents are most pronounced near bubbles at a volume resonance and are located along solid boundaries, thus contributing to the cleaning process. Micro-streaming can survive several stable regimes, as the amplitude changes and can develop higher velocities (e.g. $60 \mathrm{~cm} / \mathrm{s}$ ) at bubble surfaces. To note, both types of acoustic streaming (inside or outside the boundary layer) enhance sonochemical reactions. Strong currents and small boundary layer thicknesses of streaming significantly enhance transport processes.

Streaming velocities can reach magnitudes of several meters per second. The direction of the streaming with respect to the solid surface depends on the kinematic viscosity of the fluid and the sound intensity. For the case of water using high sound intensity (particle velocity amplitude of the bubble surface is $31-60 \mathrm{~cm} / \mathrm{sec}$ ) streaming will be directed toward the surface ${ }^{[65]}$ This streaming appears as a jet directed toward the surface that removes contaminants without causing any surface damage.

The cleaning effect of acoustic cavitation can be experimentally confirmed during 2 hours of fouling with calcium without and with ultrasonic treatment (Figure 10). In the absence of ultrasound a membrane surface develops a continuous fouling layer and covers the entire area (Figure 10B). In contrast, ultrasound causes pore deblocking, but also forms few patches of foulants at lower concentration of calcium (Figure $10 \mathrm{C})$. Although acoustic cavitation nearly prevents the cake fouling the permeate flux can be only as high as $70 \%$ of the clean water flux. This could be contributed by an increase in hydraulic resistance due to partly blocking of the inner membrane pores as a result of deposition of particulates. To note, the membrane pores subjected to cavitation do not undergo enlargement or damage (Figure $10 \mathrm{C}$ and E). Overall, the use of ultrasound acts to lower the compressibility of both the initial fouling deposit and the growing cake. The pore blockage parameter is not significantly affected. The mechanisms involved in the flux enhancement are not clarified, as the role of cavitation and acoustic streaming alone or in combination is not clear.

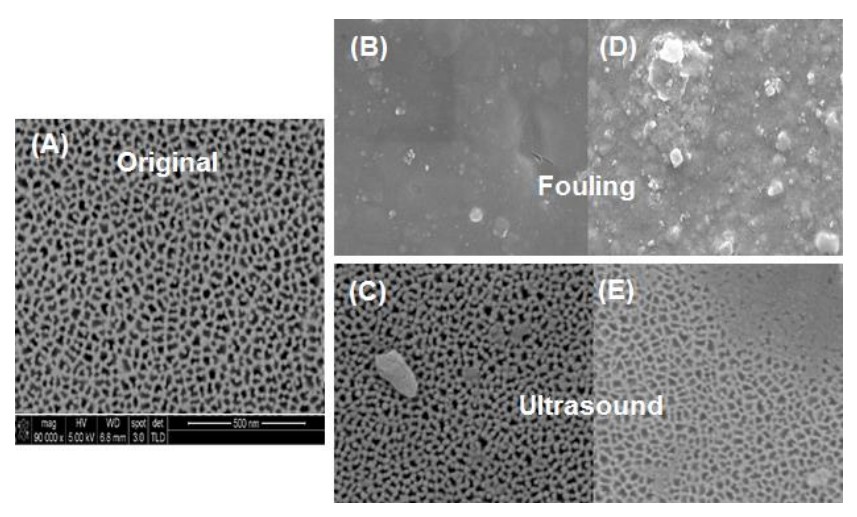

Figure 10. Figure Caption. SEM images of a membrane surface $A$ ) original and after 2 hours of fouling with calcium $(40 \mathrm{mg} / \mathrm{L}) \mathrm{B}$ ) without and C) with ultrasonic treatment, after 2 hours of fouling with calcium $(180 \mathrm{mg} / \mathrm{L}) \mathrm{D}$ ) without and E) with ultrasonic treatment. The scale bar $(500 \mathrm{~nm})$ in A) applies to each SEM image in B-E. Reprinted with permission from the reference $Y$. Gao, D. Chen, L. K. Weavers, H. W. Walker, Journal of Membrane Science 401- 402 (2012) 232- 240

Although the effective removal of microparticles from substrates by ultrasound is an established technique ${ }^{[71]}$ the physical mechanism is not well understood. Experimentally the removal efficiency of particulates by ultrasound increases with longer treatment and higher temperature of a solution. The ultrasonic efficiency is lower, if the particles have diameters smaller than $0.3 \mu \mathrm{m}$ and the acoustic pressure field has higher frequency and lower power. Alternatively acoustic cavitation at very high frequency $(e . g . \mathrm{GHz})$ can form the pressure gradient across the particle and remove it from a surface. In this mechanism cavitation collapse is eliminated and the pressure force can act as the driving force for detachment of particles. ${ }^{[62]}$

According to the line-lifting mechanism cavitation bubbles can attack the edge of the gap between the contaminant film and the surface ${ }^{[63]}$ (Figure 11). They can penetrate between the solid surface and the edge of the film and reside under the edge of the film (Figure 11A). As the bubble vibrates, a force $F$ begins to act upon the film and tear it away into the bulk water (or from the water surface). As the film separates, the bubbles slide in the direction of the untouched part of the metal and penetrate under it (Figure 11B). This process continues until the entire film has been separated from the surface. The line lifting can be eliminated by a frequency sweep. The removal efficiency increases with increasing particle radius and an initially high 
particle concentration. It is important to emphasize, that the Saffman lift force in the detachment is stronger on larger particles due to larger separation distance or a change in the contact area. $^{[64]}$ On the other hand particles with a submicrometer dimension can be effectively removed by rotational rinsing in an acoustic field. This effect is more pronounced due to wall shear stress in the viscous sublayer of the fluid, where the contaminant particles reside. Longer residence time due to adhesion-induced deformation of particles on the surface lowers the removal efficiency.

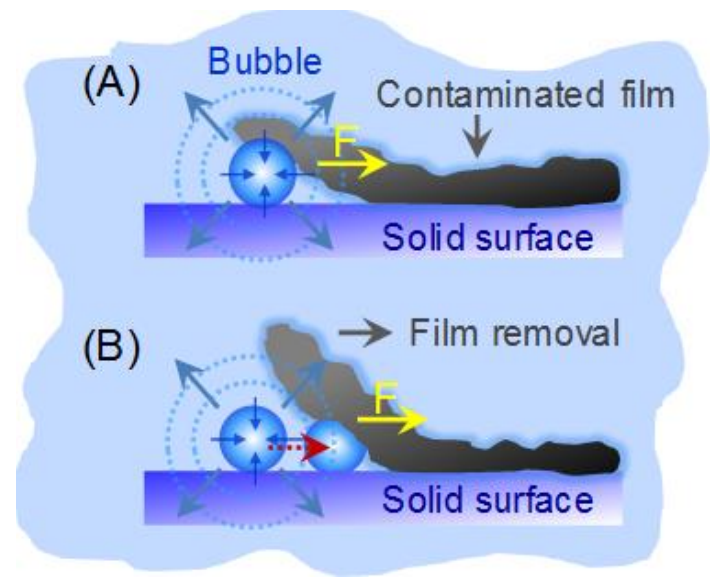

Figure 11. Figure Caption. Schematic illustration of the line lifting mechanism with the explanation of the physical principle of ultrasonic cleaning of a solid contaminated surface. A) A cavitation bubble can attack the edge of the gap between the contaminated film and the surface through the penetration between the solid surface and the edge of the film. As the bubble vibrates, a force $F$ begins to act upon the film and tear it away. B) As the film separates, the bubble slides in the direction of the untouched part of the solid surface and penetrates under the film (Figure $5 \mathrm{~B}$ ). This process continues until the entire film has been separated from the surface. Adapted from the reference Rozenberg, L., "On the Physics of Ultrasonic Cleaning," Ultrasonic News, 1960, 16-20.

In addition to the gradual peeling of pieces of the contaminated (fouled) film (higher frequency), ultrasonic crumbling can be advanced by shock waves at bubble collapse (lower frequency) and accumulation of bubbles at surface inhomogeneities. If acoustic streaming decreases the boundary layer thickness (higher frequency), the removal and transportation of smaller contaminant species increases. The removal efficiency can be monitored by the measurement of the electrical potential, which is lower on a cleaner surface.

Overall, the physical mechanism of cleaning by bubbles relies on the acoustic pressure gradient $(\Delta P)$, which causes the bubble to oscillate and radiate a sound field (Scheme 1). Objects, which are within this sound field experience a secondary radiation force, ${ }^{[72]}$ which has greater values at the bubble's wall. ${ }^{[9]}$ On the other hand, an oscillating bubble can form a streaming flow around it ${ }^{[73]}$ with a drag force, which forms flow patterns ${ }^{[74]}$ Distinct from attractive or repulsive secondary radiation forces, a streaming flow can transport particulates in an orbiting pattern around the oscillating bubble. In this way, the transport of smaller particulates can be determined by micro- streaming, while larger species will undergo the action of a secondary radiation.

At present most of the studies in ultrasonic antifouling are limited to the pore deblocking rather from the contaminated surface layer than from the inner membrane structure. There is no evidence that ultrasound can remove fouled layers from the internal membrane walls by micro-jets, shock waves or different types of streaming (Scheme 1). The question is how to enhance the length of micro-jets and their selectivity in order to increase the penetration depth and removal of contaminants.

On the other hand, it is known that ultrasound can cause fine grain refinement within the solid structure, which can be attributed to heterogeneous nucleation and acoustic streaming. ${ }^{[75]}$ Two possible mechanisms, which are the cavitation-induced dendrite fragmentation and the cavitationenhanced nucleation, ${ }^{[76]}$ can determine the properties of grain refinement. According to the former, the shock waves, which are produced by the collapse of bubbles, can result in dendrite fragmentation and promote nucleation events. ${ }^{[7]}$ In the latter cavitation enhances the wettability of impurities, which enables the impurities to act as nucleation sites. ${ }^{[78]}$ At present these mechanisms are not fully understood.

Ultrasonic treatment can suppress the dendritic inner structure, but significantly refine globular grains without homogeneous distribution, but larger clustering. ${ }^{[79]}$ The latter can be avoided, if ultrasonic treatment occurs at isothermal conditions. Ultrasonic treatment causes also particle movement (e.g. SiC) to the peripheral and the top parts of a melt due to acoustic streaming. The moved particles tend to accumulate at the peripheral part of the melt due to friction of the wall and the centrifugal effect of the vortex. Clustering can occur at the top of the melt owing to their lower density than in the matrix melt. If a solid contains dissolved gas, ultrasound creates degassing and dispersion of small particles followed by the formation of nanostructures. This process is accompanied by a particulate reinforcement of metal matrix composites during solidification and melting. ${ }^{[80]}$

\subsection{Methods in Antifouling of Biofilm}

\section{a) Structure and Composition of a Biofilm}

In general, a fouling biofilm consists of microorganisms, which live in a self-produced matrix of hydrated extracellular polymeric substances (EPS), that form their immediate environment. ${ }^{[81]}$ Such substances are mainly polysaccharides, proteins, nucleic acids and lipids. The latter provide the mechanical stability of biofilms, mediate their adhesion to surfaces and form a cohesive, three-dimensional polymer network. In addition, the biofilm matrix acts as an external digestive system by keeping extracellular enzymes close to the cells, enabling them to metabolize dissolved, colloidal and solid biopolymers. For this reason the biofilm contamination can occur on the external and internal walls of the membrane structure.

Water is the major component of the matrix, which provides a highly hydrated environment with a slower drying rate than its surroundings. Many extracellular polymeric substances are hygroscopic with a strong affinity to retain water entropically rather than through specific water binding mechanisms. For 
example, hydraulic decoupling during rapid wetting or drying events may protect the biofilm-embedded bacteria in unsaturated soils. ${ }^{[82]}$

The EPS matrix can act as a molecular sieve, sequestering cations, anions, apolar compounds and particles from an aqueous phase. ${ }^{[83]}$ The EPS contains apolar regions, groups with hydrogen-bonding potential, anionic groups (e.g. in uronic acids and proteins) and cationic groups (e.g. in amino sugars). ${ }^{[8]}$ The surface of the matrix acquires a coating with more adhesive properties, which enhances the adsorption and accumulation of particles. In this way heavy metals such as $\mathrm{Zn}^{2+}$, $\mathrm{Cd}^{2+}$, and $\mathrm{Ni}^{2+}$ bind to cell walls of bacteria in activated sludge, whereas hydrophobic compounds such as benzene, toluene and xylene can be present in the matrix. ${ }^{[85]}$ The response of biofilms to absorbed substances can be complex, e.g. toluene induces enhanced production of carboxylic groups in $P$. putida biofilms. ${ }^{[8]}$

\section{b) Antifouling of Biofilm}

Anti-fouling can be carried out by overcoming the cohesive and adhesive forces of the matrix. ${ }^{[81]}$ To note, the cohesion of flocs and biofilms determines the stability of several important processes, including flocculation, settling and dewatering. ${ }^{[87]}$ The mechanical properties of biofilms can be influenced by shear forces, ${ }^{[88]}$ which are involved in the rolling of bacterial microcolonies along surfaces. ${ }^{[89]}$

As biofilms display viscoelastic properties with reversible elastic responses and irreversible deformation, they may act as a viscous fluid. ${ }^{[90]}$ Higher viscosity is due to the fluctuating binding between the EPS matrix components. The latter can be kept together by weak physicochemical mechanisms via hydrogen bonds, van der Waals forces and electrostatic interactions. Higher mechanical stability of a biofilm can result from $\mathrm{Ca}^{2+}$-mediated crosslinking of polyanionic alginate molecules.

The common method to mitigate biofouling in reverse osmosis desalination is continuous oxidation by chlorine, combined with sodium bisulfate dosing of the feed. In contrast to ultrasonic treatment most reverse osmosis membranes are thin film composite polyamide membranes, which can be oxidized by chlorine ${ }^{\left[{ }^{191]}\right.}$ At present there is little to no knowledge about the ultrasonic action and possible mechanisms of removal of biofouled films on membranes.

\section{c) Ultrasonic Detection of Biofouling}

Few studies have been carried out to investigate the biofouling process in membrane operation by using ultrasound. Ultrasonic noninvasive real-time detection can be considered as part of a time domain reflectometry method. ${ }^{[92]}$ To note, biofouling is a phenomenon, which involves aggregates of microbes at a liquid/solid interface, that are encased in a matrix of highly hydrated extracellular polymeric substances. ${ }^{[93]}$ As the difference in acoustic properties between a biofouling layer and water or a membrane is small, it requires a periodic doping by an 'acoustic enhancer' (e.g. colloidal silica). In this way, biofouling can be detected on flat sheet polyethersulfone ultrafiltration and thin film composite polyamide reverse osmosis membranes in a canary cell flow configuration.

\subsection{How to Prevent Fouling?}

The membrane fouling can be prevented or reduced by addition of flux enhancers and nanomaterials or through optimized hydrodynamics or inhibition processes. ${ }^{\text {[94] }}$ For example, a cationic polymer can entrap soluble foulants in the bulk phase during the flocculation process ${ }^{[95]}$ and can lead to a more porous biofilm enhancing filtration. Powdered activated carbon, polyaluminum chloride or ferric salts enhance filtration in the production of potable water and reduce fouling. Higher concentration of ferric hydroxide in a precoating of the membrane can decrease the $\mathrm{pH}$ value and reduce the microbiological activity. In this way, removal of a phosphorus substance can be improved and filtration resistance can be increased.

In another method, the amount in the sludge supernatant can be reduced in the presence of salts or polymers such as $\mathrm{FeCl}_{3}, \mathrm{PACl}$, chitosan, and starch. ${ }^{[95]}$ The presence of fullerene $\mathrm{C}_{60}$ in a coating of ceramic membranes or in the form of colloidal aggregates in suspension can inhibit the respiratory activity and attachment of bacteria due to an increased hydrophobicity. ${ }^{[96]}$ The addition of silver, copper-based, magnesium or titanium oxide nanoparticles can improve anti-microbial and anti-fouling effects. The presence of gaseous bubbles can reduce the air requirements by increasing the bubble diameter. ${ }^{\left[{ }^{[7]}\right.}$ At a distance closer to the sparging clouds of bubbles the suction point can be larger, and the biocake porosity can be lower. The presence of polyester-urethane sponges increases the permeability and the effluent quality. ${ }^{[88]} \mathrm{A}$ novel strategy to control the fouling of a membrane can be an inhibition of bacterial communication by deactivating of single molecules (e.g. $\mathrm{N}$-acyl homoserin lactone), which could initiate biofilm formation. ${ }^{[99]}$

Four filtration models, which have originally been developed for dead end filtration, can describe the initial flux decline. ${ }^{[100]}$ All models imply a dependence of flux decline on the ratio of particle size to pore diameter. Among them the standard blocking and cake filtration models could be used to predict the initial flux decline during colloid filtration ${ }^{[101]}$ or protein filtration. ${ }^{[102]}$ Back transport models such as inertial migration, shear-induced diffusion, and surface transport could also contribute to the prediction of the flux behavior in membranes. In these models the key aspect relies on the implication of the influence of particle diameter and recirculation rate, or cross flow velocity on flux. Both shear-induced and inertial lift models can predict more effective fouling removal in the presence of small particles and/or large differences between back-transport and permeate velocities. These models allow prediction of the minimum dimensions of compounds, which can likely be deposited under specific flow conditions. To note, hydrodynamic factors should be also considered, as they have a profound effect on the initial flux decline. In the latter smaller particles develop a smaller back transport velocity and cause the exponential flux decline. ${ }^{[103]}$

As another strategy, pretreatment can alter the physicochemical and/or biological properties of the feed water with less fouling formation and improved desalination performance. The efficiency of pretreatment depends on temperature, composition of a solution, properties of foulant (inorganic, organic or 
biological) and the characteristics of a membrane. ${ }^{[104]}$ As first step it should be applied to the feed solution in order to remove macroparticles and microorganisms. Organic fouling can be controlled by the hydrophilization of the membrane surface. ${ }^{[105]}$ For example, by coating a PTFE membrane with sodium alginate hydrogel, the adsorption of citrus oil on the membrane surface can be significantly decreased. ${ }^{[106]}$

\section{Ultrasonic Enhancement of Flow Filtration}

Flow filtration can be enhanced by an additional turbulence, which is induced across the membrane surface through the assistance of vibrational shear forces (shear-enhanced processing), rotation (by employing disks), additional flow with pulsation, vortex mixing, unsteady jet, two-phase liquid flow or backflushing and also by applying electric field (e.g. for ionic foulants). ${ }^{[61]}$ Backflushing or backwasing can be effective for tubular and hollow fiber membrane filters, but may not be applied to spirally wound modules. The latter can also interrupt the continuous filtration leading to a longer processing time. ${ }^{[61]}$ On the other hand, the physical effects of cavitation can be more pronounced in fluid flow conditions as it was postulated many years ago. ${ }^{[107]}$

Filtration and dewatering by ultrasound has been carried out mainly with small laboratory-scale batch or continuously operating devices in combination with an electric field or with cleaning reagents. Applications include electro-acoustic dewatering press and filters such as polishing 'CERTUS', screening 'Scamsonic' or ceramic capillary 'CERAMEC'. Ultrasound assisted cleaning could be improved, if the erosion of transducers is reduced and the transformation of electric to mechanical energy would be more efficient. In this way, research efforts should be focused on the study of material properties of filters (e.g. ceramic), engineering aspects of transducer units at different ultrasonic parameters. For example, an impedance analyzer is a good tool to check the electrical properties of the individual transducer elements and transducer boxes.

The performance by ultrasound can be improved if the sound source is located closer to the membrane surface. ${ }^{[61]}$ Greater efficiency of the fluid flow can be acquired by an ultrasonic horn with spherical geometry. ${ }^{[108]}$ In this way, radial arrays of ultrasonic transducers can generate acoustic field with a stronger focus at the center of a pipe yielding a more even acoustic field with intensity comparable to a standard horn, but with a weaker field immediately adjacent to the pipe surface. By this approach the metal abrasion of the transducer surface can be reduced. On the other hand, disintegration of bubbles into a cluster of daughter droplets during acoustic cavitation can also undergo a sequence of repetitive implosions followed by the formation of microjets with a length larger than $100 \mu \mathrm{m} .{ }^{[108]}$

A new design of a multi-stepped cone-shaped tip of a transducer can significantly increase the energy-emitting surface area and create multiple reactive zones in acoustic cavitation. ${ }^{[106]}$ In contrast to conventional horn-type transducers the new design allows higher energy efficiency $(\sim 31 \%)$ and larger rate of hydroxyl radical formation yielding faster degradation of contaminants. To keep the filtration rate on a high level ultrasonic cleaning of filter elements requires the development of new methods for in situ measurement during filtration.

\subsection{In Polymeric Membrane Filters}

Polymeric membranes have been used for ultrafiltration long before the development of ceramic membranes, e.g. a dialysis membrane, which is widely used in colloid science. Ultrasound can increase the steady-state permeation rate through the dialysis membrane by a few tens percent. ${ }^{[107}$ Changes of the solute concentration on the receiving side could be explained by a thinning and developing of the liquid film adjacent to the membrane surface during ultrasonic treatment. The solute transfer by ultrasound is reversible without damage of the membrane structure. The solute permeation is higher at a slower rate of the liquid flow near the membrane surface and with a standing wave in the vicinity of the membrane. To note, faster flow can disturb the standing wave decreasing the solute permeation. The ultrasonically enhanced mass transfer coefficient is higher at a slower flow rate than at faster flow. In this way ultrasound causes a stepwise change of solute concentration on the receiving side and an enhancement of the steady-state permeation rate. About $28 \%$ increase of steadystate permeation rate can be observed with ultrasound. At higher molecular weight of solute the degree of enhancement decreases.

The interaction of ultrasound with other types of polymeric membranes (e.g. polyethersulfone (PES), polyvinylidenefluoride (PVDF) and polyacrylonitrile (PAN)) depends on the molecular structure, interconnection and binding properties of the material. ${ }^{[109]}$ For example, higher surface area of only PES can be achieved by ultrasound, whereas the other two materials acquire less damage in the central section and little changes at the edges. Ultrasonic degradation of the membrane surface leads to an increase of the pore radius, density and porosity followed by the formation of larger cracks at the edges of the membrane. The latter can be due to the interconnection of neighboring pores. In this way, larger cracks could increase the permeability of the membrane.

Acoustic streaming and cavitation can alter the surface of a porous structure through micro-jets and shock waves impacting on the surface (Scheme 1). Ultrasonic modification of the surface of polymeric membranes can lead to an increase in pore radius and to an overall increase in pore density and porosity (Scheme 1). However, these modifications can be much less important for PVDF polymeric supports. Ultrasonically treated PVDF support acquires slightly larger pore density and porosity than the non-treated (original) membrane. The pore filling can be increased due to acoustic streaming and cavitation, which enable transport of the organic solution of the extractant through the membrane. As a consequence, the fluid decreases its viscosity due to elevated temperature and high speed liquid jets.

In spite of their great efficiency in enhancing filtration processes ultrasonic treatment causes heating and structural changes during interaction with a membrane. The overall 
temperature increase due to the absorption of sound waves or to the adiabatic compression of the medium in the sound field is dependent on the nature of the medium and the liquid/membrane interface. The structural properties (e.g. viscosity) depend on the dynamic agitation and shear stress, as ultrasound is absorbed by a solid medium yielding 'fracture' of a dense material. Ultrasonic degradation of polymers can be theoretically explained by frictional forces, shear gradients and impacts due to the cavitation collapse. On the other hand, in a new polymeric material such as an ion-exchange hollow fiber the $\mathrm{Na}^{+}-\mathrm{H}^{+}$ion-exchange can be enhanced with increasing power of the ultrasound. ${ }^{[110]}$ However, online ultrasonic irradiation has lower efficiency and higher cost, which limits its application today.

\subsection{In Ceramic Membrane Filters}

The main advantages of ceramic filters are very low energy consumption, dry filter cake, particle free filtrate, high filtration rates simple installation, operation and maintenance, integrated filter and ancillary system, continuous operation, and high availability. Ceramic filters are better suited for dewatering of slurries with high and consistent concentration of solids having the major part of the particles in the size range 30-150 microns. To note, the ceramic filter technology is used widely for dewatering base metal concentrates (copper, nickel, zinc, lead and pyrite), ferro chrome and iron ore products. In most of the ceramic filter membranes ultrasound is used at lower frequency $(20-300 \mathrm{kHz})$ due to its greater efficiency in the removal of microscopic contaminants. Ultrasound at higher frequency (400$1000 \mathrm{kHz}$ ) favors removal of contaminants at a submicroscopic scale. High frequency ultrasound efficiently cleans surfaces from microscopic particles due to acoustic streaming at a larger scale movement of fluid. The ultrasonic performance is often combined with back flushing and a filtrate or by washing of chemical solutions. Back flushing removes the residual cake and cleans the microporous structure and can be automatic and adjustable for each application

One of the strategies in the advancement of ceramic membranes is to increase the permeation flux and the feed flow rate by application of vibrations, sponge ball or electric field to ultrafiltration in colloidal solution. In the latter charged colloids can be forced to move away from the membrane surface to the bulk of the liquid solution by electrophoresis. To note, this process requires an appropriate $\mathrm{pH}$ with a reflection coefficient of the colloidal solution being dependent in the Reynolds number, the strength of the electrical field and the concentration of the diffusing particles. If the colloidal solution contains charged proteins with low electrical conduction, the ultrafiltration efficiency becomes higher in the electric field. In the presence of a dissolved gas gel formation can be decreased, if gas is supplied from the bottom of the membrane module, increasing the permeation ability of the latter. For example, ultrasound can increase the permeation flux in the ovalbumin solution, which is mainly determined by the pressure gradient across the membrane. This ultrasonic effect is stronger at smaller concentration of ovalbumin.
To note, in the presence of additives, e.g. dextran or polyvinyl alcohol (PVA), a gel layer growth on a ceramic membrane surface can be inhibited. In dextran solution the permeation flux rate is larger by several times than one for ovalbumin solution and is higher at a larger pressure gradient. In the presence of PVA the permeation efficiency is nearly the same as in the dextran solution. In this way, the gel polarization model can explain the permeation flux in the presence of proteins, while the concentration polarization model can be applied to the solution of dextran or PVA. In particular, for the ovalbumin solution above a critical permeation flux a gel layer forms on the surface of the ceramic membrane independent from concentration. The gel layer can be more easily taken off from the surface of the ceramic membrane by ultrasonic irradiation, if the layer is thinner. Other possible mechanisms involve osmotic pressure and resistance due to solute adsorption. In this way the concentration on the membrane surface can grow due to concentration polarization in the ultrafiltration, then osmotic pressure resists to permeation. Although ultrasonic treatment of ceramic membranes increases the mass transfer coefficient of water across membranes, it is less effective at removing fouling material inside pores. ${ }^{[111]}$ Greater improvement of the permeate flux and rejection ratio can be achieved at higher ultrasonic power (> $20 \mathrm{~W})$.

\subsection{In Liquid Membrane Systems}

\section{a) Supported Liquid Membranes (SLM)}

Overall, supported liquid membranes can be divided into the following groups: i) passive membranes (porous, solubility and ion-exchange) and ii) active (liquid and biological). ${ }^{[12]}$ The solution, which initially contains all the ions, which can permeate the liquid membrane, is generally referred to as the feed solution. The solution present on the opposite side of the membrane, which is initially free from permeable ions, is generally referred to as the strip solution. In principle, liquid membrane permeation is a solvent extraction process, in which the extraction and stripping operations are performed simultaneously. Typically the driving force is a concentration gradient of the ion-carrier complex between both sides of the membrane.

In general, the supported liquid membrane (SLM) technique uses a microporous hydrophobic polymer structure as support for the organic phase incorporating the carrier. The liquid phase is held in the membrane pores by capillarity. The wetting pressure for a hydrophobic membrane is directly proportional to the surface tension at the aqueous/organic phase and inversely proportional to the pore diameter. The surface functional groups of membranes can significantly decrease the wetting pressure (e.g. amine compounds). An ideal porous support structure requires higher porosity, smaller pore diameters, thinner walls, hydrophobic materials and chemically resistant polymer in the frame of lower costs. Porous support membranes are available as flat sheets or hollow fibers. The latter are preferred because of their higher packing densities and favorable hydrodynamics.

Overall, greater flux can be achieved by thinner membrane and lower viscosity of a liquid membrane. Higher efficiency of a membrane indicates lower selectivity and permeability of the fast 
permeating component. The SLM technique was originally developed for hydrometallurgical applications. Competing technologies in this field of application are solvent extraction, ion-exchanger and chemical precipitation. Chemical precipitation is primarily appropriate for high metal ion concentrations, but does not allow the metal to be directly recovered in elementary form. lon-exchangers have a lower selectivity for metal ions with the same charge and are mainly used, when there are very low concentrations of metallic ions in the feed solution.

At present the studies with ultrasound are limited to the preparation of supported liquid membranes. ${ }^{[113]}$

b) Liquid Surfactant Membrane (LSM)

Heavy metals and amino acids can be effectively separated and recovered by a liquid surfactant membrane (LSM) process. ${ }^{[114]}$ It is used for concentration and separation of various species from dilute solutions including metals, weak acids/bases, biologically important compounds, hydrocarbons, and gas mixtures. ${ }^{[115]}$ Ultrasound $(20 \mathrm{kHz})$ can easily produce and disperse water/oil emulsions (e.g. water/kerosene) within 1 min with a droplet diameter decreasing up to $50 \mathrm{~nm}$ at higher concentration of surfactant (in aqueous phase) and ultrasonic power.

Stable liquid membranes can be formed by impregnating commercial porous polytetrafluoroethylene or polypropylene membranes with solutions of commercial chelating extractants. ${ }^{[116]}$ When such membranes are placed between aqueous solutions of copper (II) sulfate at $\mathrm{pH} \mathrm{1-4} \mathrm{and} \mathrm{of} \mathrm{sulfuric}$ acid at pH 0.5, transfer of copper (II) ions from the less acidic to the more acidic solution took place simultaneously with an equivalent proton transfer in the opposite direction. The copper (II) ion can move selectively with respect to iron(III) ion and against a twenty-fold concentration gradient. These membranes can be regenerated by washing the porous support with a solvent and applying a fresh solution of extractant.

\section{c) Liquid Emulsion Membrane (LEM)}

Another approach is introduced by a liquid emulsion membrane (LEM) process to remove $\mathrm{Cu}$ (II) ions from aqueous solution in a water-in-oil-in-water system by ultrasound. ${ }^{[117]}$ Nearly all of the $\mathrm{Cu}$ (II) ions present in the continuous phase can be extracted within a few minutes. Such a membrane is stable at an equal volume ratio of organic/aqueous internal phase. To note, acoustic emulsification can occur easier in hexane as an organic phase, due to its lower viscosity compared to heptane and dodecane, in agreement with the physico-chemical mechanism. ${ }^{[118]}$ To note, the mechanism of droplet impact on membrane disruption is not fully understood. Application of liquid emulsion membranes (LEM) can be extended to successful filtration of organic acids, amino acids and antibiotics. ${ }^{[119]}$

The interior phase of LEM can consist of a concentrated inorganic salt solution, which provides the driving force for transport. If the carrier is initially complexed with the counterion in the interior phase, the carrier/counterion complex adsorbs to the globule/exterior phase interface. This reaction can be driven by the solute's affinity for the carrier and the oil phase. The carrier/solute complex can diffuse across the membrane until it reaches an oil/interior droplet interface. At this interface the solute is exchanged for the counterion in the interior phase followed by a back diffusion of the carrier/counterion complex across the membrane.

LEMs cannot be affected by the presence of either live bacterial cells or enzymes, suggesting, that fermentation broths could be handled without pretreatment. ${ }^{[120]}$ However, the membrane of an LEM may break due to agitation-produced shear, excessive internal droplet size or a weak membrane structure. The latter can be avoided by increasing the viscosity of the membrane,$^{[121]}$ the concentration ${ }^{[122]}$ or by the type of surfactant or phase ratio of the membrane.

At present the application of ultrasound is restricted to the membrane formation through emulsification to acquire advanced properties in extraction of toxic ions (e.g. arsenic V) from an aqueous medium. ${ }^{[123]}$ The extraction of arsenic is stronger, if the diameter of emulsion droplets is smaller, in agreement with the physico-chemical mechanism of acoustic emulsification. ${ }^{[118]}$ In an acoustic field an internal droplet size can be initially decreased and then increased by increasing the concentration of surfactant up to a certain value (e.g. Span 80). Increasing the concentration of Tween 20 as a hydrophilic emulsifier up to an optimum concentration decreases the internal droplet size and increases the extraction amount. By increasing the sonication time up to $4 \mathrm{~min}$, the internal droplet size is decreased and the extraction amount is increased. If the sonication time is increased further, the internal droplet size is increased and the extraction amount is decreased.

\section{Cross Flow Filtration by Ultrasound}

A cross flow filtration can be generally conducted at low transmembrane pressures and a high cross flow velocity, which tend to loosen and remove the contaminating fouling layer Cavitation effects are stronger at lower pressure, and the higher cross flow velocity does not decrease the ultrasonic efficiency. The intrinsic permeability and structure of the membranes can or cannot be altered by ultrasound. Ultrasound may not alter the feed solution, and its efficiency is independent on the particle radius (e.g. silica particles). ${ }^{[124]}$ To note, the efficiency of ultrasonic cleaning alone and in combination with forward flushing can be as high as $87 \%$ and $98 \%$ (87). In cross flow filtration the ultrasonic effect may be independent on the particle size. ${ }^{[61]}$ On the other hand, ultrasonic-assisted cross flow cleaning may rely on the detachment of particles from a membrane surface by micro-streaming and turbulence.

The ultrasonic filtration of inorganic contaminants (e.g. nylon microfiltration membranes fouled by Kraft paper mill effluent) can be increased at a lower bulk temperature. On the other hand, cleaning of membranes from proteinaceous fouling is more effective at higher bulk temperature. Ultrasound at higher power (e.g. 0.21 to $83 \mathrm{~W} / \mathrm{cm}^{2}$ ) and with lower frequency $(20 \mathrm{kHz}-50 \mathrm{kHz})^{[125]}$ enhances the cleaning efficiency to a greater extent. Without altering the feed solution ultrasound can also maintain the intrinsic permeability and structure of the membranes while operating at higher efficiency. In contrast to continuous mode a pulsed mode of ultrasonic treatment is more 
effective in maintaining higher flux and permeability of BSA in cross flow ultrafiltration. ${ }^{[126]}$

The filtration pressure affects both the cavitation properties of ultrasound and the deposition behavior on the membrane surface. Lower transmembrane pressures and higher cross flow velocity tend to loosen and remove the fouling cake without decreasing the ultrasonic efficiency. As discussed above, as pressure increases, fewer acoustic bubbles form, but collapse more violently. On the other hand, the permeate flux can be improved with a higher applied transmembrane pressure ${ }^{[127]}$ and cause an increase in cavitation effects and hydrodynamic turbulence. The relationship between flux and power may be nonlinear in the higher power range due to the conversion of ultrasonic energy into heating.

Cross flow microfiltration can be explained in the frame of a shear-induced diffusion model. ${ }^{[128]}$ According to this model, the fouling layer thickness increases at higher concentration of particulates and transmembrane pressure drop, but decrease the shear rate. Initial rates of the particle layer buildup agree well with the theoretical predictions and are essentially the same as those for dead-end filtration. In this model an inertial lift may be the predominant mechanism of particle motion away from a membrane surface with a lift velocity depending on the channel Reynolds number. ${ }^{[129]}$

\subsection{Acoustic Approaches without Electric Field}

One type of approaches is to assemble an ultrasonic transducer into the membrane module in order to perform ultrasonic online cross flow filtration of industrial wastewater. ${ }^{[128]}$ If ultrasound propagates from the feed flow side, the membrane structure becomes more open and can be easily plugged by contaminated species. If the membrane has a compact density, the ultrasound irradiated from the feed side of the membrane significantly increases the flux. At higher ultrasonic intensity the membrane surface gradually develops erosion spots. Ultrasonic treatment at atmospheric pressure during a short intermission pause in filtration can be another efficient method in membrane filtration. Lower frequency (e.g. $27 \mathrm{kHz}$ not at $200 \mathrm{kHz}$ ) of ultrasound significantly improves the flux due to violent turbulence and streaming, which are intensified by liquid jets. In contrast, ultrasound at higher frequency increases flux at milder conditions due to streaming without damaging effect

Another type of approaches involves the principles of micro-fluidic flows in the presence of ultrasound or acoustic cavitation. For example, acoustophoresis can effectively separate particles by acoustic radiation forces. ${ }^{[129]}$ The acoustophoretic separation can be increased, if particles have larger differences in diameter and density. ${ }^{[130]}$ Acoustophoretic actuation requires acoustic pressure wavelengths comparable to the size of particles and/or the channel dimensions. For typical microfluidic dimensions (10 to $100 \mu \mathrm{m}$ ), the acoustic frequency is in the range of $1 \mathrm{MHz}$ to $100 \mathrm{MHz}$. In the $\mathrm{MHz}$ range for cleaning of organic solutions this method may require external cooling in order to minimize unnecessary heating. ${ }^{[131]}$ However, this can be avoided, if ultrasound has a lower frequency $(<100$ $\mathrm{kHz}$ ).
In another method, when a bubble is excited by the piezoactuator near its resonance frequency, the oscillating bubble can capture microparticles by an attraction force. The bubble mobility can be actuated in cavitation micro-streaming flow through the agitation of the surrounding fluid in a small chamber. ${ }^{[132]}$ When the particles arrive at the desired location, they can be released from the bubble by turning off the piezo-actuator excitation. In this way a mobile oscillating bubble can selectively trap and separate particles. Oscillating bubbles can be individually trapped in a bubble-based microfluidic device and form acoustic streaming in order to enhance the mass transfer in the liquid/liquid extraction process. ${ }^{[133]}$ At a frequency of $10 \mathrm{kHz}$ the maximum amplitude of the bubble oscillation can reach values of up to $6 \mu \mathrm{m}$. In general, the bubble oscillations are governed by interactions between acoustic waves, gravity and capillary waves. It is assumed, that the bubble frequency response can be dominated by capillary forces.

Another approach introduces the platform with surface acoustic waves, which act on droplets residing on a hydrophobic surface. The microfluidic unit operations can be controlled by acoustic shock waves travelling on the surface of the support. The shock waves can be generated by an arrangement of surrounding sonotrodes and define the droplet manipulation area. To note, the amplitudes of these waves are only few $\mathrm{nm}$. Most of the unit operations such as droplet generation, transport or mixing can be freely programmable. ${ }^{[134]}$ This approach also allows handling of small liquid volumes (nanoliter) in droplets on planar surfaces. The transport mechanism by surface acoustic waves becomes more flexible, since it depends only on the viscosity and surface tension of the liquid. It has long term stability, but complexity of hydrophobic and hydrophilic surface coatings. For this reason it requires higher costs for the disposable chip and the instrument.

Acoustically driven microbubbles can induce microscale hydrodynamic flows, which are used in fluid pumping, ${ }^{[135]}$ mixing ${ }^{[136]}$ or particle collection. ${ }^{[137]}$ The superposition of bubble streaming and Poiseuille flows can form a closed upstream vortex, which can trap larger particles, followed by release from a cluster. By this approach particles with different dimensions can be separated into various outlets due to the narrowly focused trajectories. If particles have different density, Bjerknes forces become more effective in addition to steady streaming flow. ${ }^{[138]}$ Attraction and repulsion between particles and bubbles can be controlled by the interplay of the drag force due to streaming flow and the Bjerknes force.

In a new design an upstream vortex for trapping of particles could be avoided as the microbubble generated a steady streaming flow by a remotely actuated acoustic pressure field (Figure 12). In this way the bubble streaming flow accelerated the flow near the bubble with a local velocity, which significantly increased the particle separation. In a conventional pinched flow fractionation (PFF) microdevice the solution with particles requires alignment onto the sidewall in the pinched segment for the separation (Figure 7A and B). Such a design cannot allow separation of particles with $2 \mu \mathrm{m}$ and $10 \mu \mathrm{m}$ diameters (Figure 7C). On the other hand, with the bubble enhanced PFF microdevice, the particles can be clearly 
separated with a twice longer distance (Figure 7D). The separation can be greatly improved at higher velocity flow of the field depending on the type of flow ${ }^{[139]}$ (low frequency - 'fountain' and high frequency 'anti-fountain') (Figure 12).

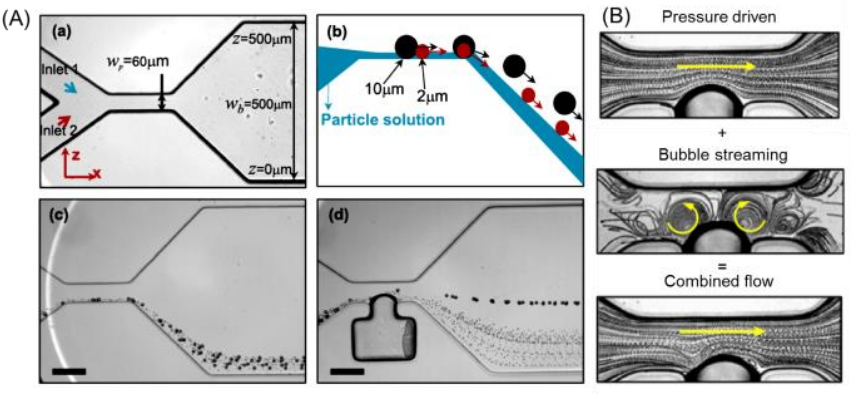

Figure 12. A) In (a) microscopic image of a conventional pinched flow fractionation device, which involves two inlets passing through a narrow neck with a width $w_{p}$ of $60 \mu \mathrm{m}$ with an end out into a broadened segment with a width $w_{b}$ of $500 \mu \mathrm{m}$. The velocity profile within the pinched segment is assumed to be linear. In (b) schematic presentation of a mechanism of separating particles with larger $(10 \mu \mathrm{m})$ and smaller $(2 \mu \mathrm{m})$ diameters. In (c) and (d) microscopic images of a pinched flow fractionation device during separation of a particle solution without (conventional) and with ultrasound. The scale bar is $100 \mu \mathrm{m}$. Reprinted with permission from the reference $\mathrm{R}$. Zhou and C. Wang, J. Micromech. Microeng. 25 (2015) 084005. B) Superposition of the flow field in the microchannel with non-vibrating bubble and acoustic streaming flow field. The combination of the 'fountain' type flow (low frequency of ultrasound, $41 \mathrm{kHz}$ ) and the pressure driven flow forms unique flow structure. The latter affects the particle trajectories and enhances the separation between particles of different diameters. Reprinted with permission from the reference R. Zhou and C. Wang, J. Micromech. Microeng. 25 (2015) 084005

The combination of the 'fountain' type flow and the pressure driven flow forms a unique flow structure, which affects the particle trajectory and enhances the particle separation. The velocity distribution of the conventional PFF has a parabolic profile and is symmetrical about the centerline. In contrast to the acoustic bubble enhanced PFF the lower half has a higher velocity, which is also much higher at a distance closer to the bubble with an interfacial oscillation. The average velocity near the lower wall surface is larger than that of the upper wall surface due to the enhanced acoustic flow of the 'fountain' type. The volume width of the particle solution decreases, as the value of the flow rate does not change. The large particles are aligned onto the bubble surface and pushed onto a streamline that is farther away from its original streamline because of the combined flow field. The acoustic bubble flow field improves the separation, as the small difference inside the pinched segment is significantly enlarged in the broadened segment. Higher driving voltage increases the separation distance between smaller and larger particles due to larger acoustic streaming flow. This is due to the direct proportionality between the value of the driving voltage and the square of the velocity in the acoustic streaming flow. ${ }^{[140]}$

\subsection{Electroacoustic Methods}

In general, in microfluidic devices the separation of microobjects from a liquid stream can be selectively performed in combination with acoustic fields, ${ }^{[141]}$ magnetic or electric forces ${ }^{[142]}$ dielectrophoresis ${ }^{[143]}$ or hydrodynamic principles. ${ }^{[144]}$
The physico-chemical mechanisms of this combination can be predicted by the pressure driven laminar flow platform. One of the strengths of such a platform is the continuous processing of samples, which allows online monitoring of water quality control or separation processes. The platform is in principle compatible with polymer mass-production technologies such as injection molding and enables inexpensive disposable microfluidic chips. A difficulty of the platform is the necessity to connect the pressure source to the (disposable) chip, which decreases the portability and requires additional manual steps.

In contrast to continuous liquid flow in microfabricated channels, digital microfluidics does not require any pumps or valves, and discrete bubbles can be independently manipulated on a two dimensional surface in an electrical field. ${ }^{[145]}$ In general, a digital microfluidics, which employs bubbles, is based on four fundamental operations: the creation, transportation, splitting, and merging of bubbles. ${ }^{[146]}$ A microfluidic device can simultaneously apply electric and ultrasonic fields in a threedimensional (3D) microelectrode scheme, where the electrodes function as both electroporation electrodes and cell flow channel. ${ }^{[147]}$ This 3D microelectrode configuration also allows a uniform electric field to be applied, while making the device compatible with fluorescence microscopy. But this method is mostly applied to the delivery of small molecules into the cellular interior maintaining higher cell viability. If the electric field and acoustic wave have perpendicular directions, transient pores along two axes of the cell membrane can be formed at lower intensities.

In general, electric field-based approaches such as electrophoresis and dielectrophoresis can be used for small object manipulation due to the difference in electric properties of the species. These are typically on-chip operations with microfabricated electrodes. ${ }^{[148]}$ A DC electric field can be applied to surface charged objects for electrophoresis while a nonuniform DC or AC field can be applied to dielectric objects for dielectrophoresis. An on-chip micro-object manipulation method uses the phenomenon of acoustically excited bubbles. The oscillation frequency of these bubbles can be in the range from 5 $\mathrm{kHz}$ to $20 \mathrm{kHz}$, which enables capturing of neighboring objects. When the oscillating bubbles are transported by electrowetting the captured objects are carried by the oscillating bubbles and released in a desired position upon turning off the acoustic excitation. In this method acoustic excitation is created by a piezo-actuator for bubble oscillation, and electrowetting is used for bubble transportation. Typically, to transport bubbles in a controlled manner, the electrowetting electrodes need to be patterned and arrayed. Microstructured arrays on the electrode surface can be acquired by using standard lithography technology. The main fabrication process on the bottom plate consists of three steps: metallization and patterning of electrodes, deposition of the dielectric layer, and deposition of the hydrophobic layer. Other methods involve the principle of electrowetting, and ultrasound is applied only to enhance the surface vibrations at the gas/liquid interface. The amplitude of these vibrations can be in the order of several tens or hundreds of $n m .^{[149]}$ 


\section{Summary and Outlook}

Among the ultrasonic parameters absorption at higher frequency can favor stronger acoustic streaming, while scattering decreases its energy. The topology of the membrane surface and the orientation of ultrasound at the interface can predict the gain or loss of ultrasonic energy. If the inhomogeneity of the membrane filter is parallel to the shear movement of streaming, the dependence on the liquid viscosity becomes larger at resonance. If they are perpendicularly oriented, liquid portions can be trapped and at a critical angle the reflected wave can be damped.

The mutual interaction of ultrasound with mechanical, electrical and fluid fields in a micro-pump system can significantly accelerate mass transport and overcome the diffusion limit. In the presence of a thermoacoustic transducer, i.e. the coupling of a heat emission wave and ultrasound, heat flux and heat capacity can dominate the thermoacoustic phenomenon neglecting heat convection. In this method mass transport can be accelerated by heating with ultrasound at higher frequency. In acoustophoresis of a deformable reflector mass transport including solids and liquids at smaller scale can be performed by acoustic forces in contactless manner. Any dense material independent from its physical properties can be transported in a fluid flow eliminating possible contamination. The mutual interaction of micro-streaming and mechanical stirring (i.e. by magnetic stirrer) can increase the velocity of fluid transport to the membrane filter, but not across its porous structure. In another concept, the laminar pattern of a fluid flow can be disturbed through pressure fluctuations of ultrasound and be strongly accelerated by bubble oscillations. The latter can be entrapped in crevices of microfluidic systems and can focus acoustic energy due to the coupling with acoustic waves. The velocity distribution of an accelerated fluid flow can be predicted through modelling of a radiator structure, which has its maximum value at the cutting edge. Electroconvection, which employs electric forces to form non-stationary currents to increase the fluid flow, can be combined with ultrasound as perspective method to direct accelerated mass flows by purpose. Overall, these methods are promising for acceleration of fluid flow, but, in a perspective, require significant technical improvement to meet the requirements at an industrial-plant scale. However, they can be successfully extended and applied in any scientific laboratory.

Close to the surface of a membrane filter, ultrasound can cause or prevent antifouling. The cleaning action of ultrasound mostly relies on micro- and acoustic streaming, which can reduce the diffusion boundary layer and remove the contaminating coating of a buildup cake or gel-like layers. As great advantage of ultrasound, this technique can involve both physical and chemical cleaning methods through acoustic cavitation. The latter can scour the surface, remove the fouling layer and reduce concentration polarization at the same time. On the other hand, it can enhance and maintain the flux due to turbulence and antifouling. But more important is its action to prevent the fouling through the agglomeration and degradation of particles in a fluid flow towards the filter. The working principle of ultrasonic antifouling can be explained by the 'line-lifting' mechanism involving bubble penetration into the pores and gaps of a contaminated layer. However, it cannot explain the removal of individual particles and not of a film. In a similar way, acoustic streaming, which is considered more influential in antifouling, cannot explain particle removal. Although streaming is effective in antifouling its action is restricted to the surface of a filter leaving the pores within a membrane structure contaminated. As acoustic streaming is derived by shock waves and micro-jets of bubble transient collapse jets may be used to clean the inner pores, but its experimental evidence is absent. As a perspective, grain refinement of inner structure and surface functionalization of the filter may make an important contribution to overcome this problem.

Ultrasonic enhancement of flow filtration in ceramic, polymeric or liquid-types of membrane filters requires new methods for in situ measurement during the fluid flow. Alternatively, future efforts should be directed into new chemical procedures for surface chemical (e.g. wettability) or morphological (e.g. larger pore radius) modification of fluid composition and membrane filter in the context of 'green chemistry' and lower costs. On the other hand, cross flow at lower transmembrane pressure, but higher cross flow velocity can be advanced by new methods with ultrasound or stable cavitation. The combination of the latter with the electric field can be selectively performed and predicted, but depends on the design of the supporting platform.

Overall, at present the choice of methods with ultrasound is limited to few small scale systems, which involve electric or thermal treatment of fluid flows and include acoustic coupling in absorption or reflection. Despite their originality these methods can be applied only to accelerate the flows in specific systems and need excessive development of the technical performance at a larger scale. From the ultrasonic side, the relationship between the frequency or intensity of ultrasound and the velocity of flow and mass transfer of complex fluid is little studied. In contrast, antifouling of membrane filters is well understood in the frame of streaming, but cannot be applied to individual particulates and inner structure. Still, little is known about the impact of shock waves and micro-jets in antifouling and their potential effects across the filter. The sonochemistry of a complex fluid and physico-chemical modification (functionalization) of a membrane filter can gain new knowledge in acoustic cavitation and its consequences for wastewater treatment.

\section{Acknowledgements}

The financial support from the Max-Planck Society and the DFG RA2111 research grant are acknowledged. $H$. M. acknowledges fruitful discussions with T. Zemb, J. Duhamet and $J$. Theisen and support from the European Research Council under the ERC Grant Agreement Nr. 320915 "REE-CYCLE": Rare Earth Element reCYCling with Low harmful Emissions. We 
also acknowledge support from DFG (G8-project SmartCoat) and the Fonds der Chemischen Industrie.

\section{Keywords: physical chemistry $\bullet$ chemical physics •} materials science $\bullet$ soft matter $\bullet$ liquids and fluids

[1] M. A. Shannon, P. W. Bohn, M. Elimelech, J. G. Georgiadis, B. J. Marinas, A. M. Mayes, Nature 2008, 452, 301-310.

[2] Y. Gao, D. Chen, L. K. Weavers, H. W. Walker, J Membr. Sci. 2012 401, 232-240.

[3] M. Y. Koh, T. I. Mohd, Renew. Sust. Energ. Rev. 2011, 15, 2240-2251.

[4] M. R. Hoffmann, I. Hua, R. Hochemer, Ultrason. Sonochem. 1996, 3, 163-172.

[5] E. Joyce, S. S. Phull, J. P. Lorimer, T. J. Mason, Ultrason. Sonochem. 2003, 10, 315-318

[6] H. Bruus, Theoretical Microfluidics, Vol. 18 (Ed.: H. Bruus), Oxford University Press, 2007, p.346.

[7] D. Peters, J. Mater. Chem. 1996, 6, 1605-1618.

[8] M. O. Lamminen, H. W. Walker, L. K. Weavers, J Membr. Sci. 2004, 237, 213-223.

[9] T. G. Leighton, The Acoustic Bubble, (Ed.: T. G. Leighton), Elsevier, 1994, p. 613.

[10] K. S. Suslick, Ultrasound; Its Chemical Physical and Biological Effects, (Ed.: K. S. Suslick), Wiley-VCH, Weinheim, 1988, pp. 123.

[11] L. D. Landau, E. M. Lifshitz, Course of Theoretical Physics, Vol. 6: Fluid Mechanics, London, 1966, p. 536

[12] R. J Wakeman, E. S. Tarleton, Chem. Eng. Res. Des. 1991, 69, 386397.

[13] M. Valverde, Soft Matter 2013, 9, 8792-8814.

[14] S. J. Lighthill, J. Sound. Vib. 1978, 61, 391-418.

[15] H. Schubert, Powder Technol. 1984, 37, 105-116.

[16] C. Soria-Hoyo, J. Valverde, A. Castellanos, Powder Technol. 2009, 196, 257-262.

[17] T. Prozorov, R. Prozorov, K. S. Suslick, J. Am. Chem. Soc. 2004, 126, 13890-13891.

[18] H. D. Liess, A. Knezevic, M. Rother, J. Muenz, Faraday Discuss. 1997, 107, 39-52.

[19] a) G. Mott, J. Acoust. Soc. Am. 1971, 50, 819-829; b) F. J. Lockett, J. Mech. Phys. Solids 1962, 10, 53-64.

[20] J. L Laborde, C. Bouyer., J. P. Caltagirone, A. Gerard, Ultrasonics 1998, 36, 589-594.

[21] T. J. Mason, Ultrasonics 1992, 30, 192-196.

[22] L. C. Hagenson, L. K. Doraiswamy, Chem. Eng. Sci. 1997, 53, 131-148.

[23] a) C. Zhu, G. Liu, J Membr. Sci. 2000, 176, 31-41; b) L. H. Thompson, L. K. Doraiswamy, Chem. Eng. Sci. 2000, 55, 3085-3090.

[24] L. A. Crum, Ultrason. Sonochem. 1995, 2, S147-S152.

[25] a) N. Riley, Annu. Rev. Fluid Mech. 2001, 33, 43-65; b) A. Gopinath, A. F. Mills, J. Heat Transfer 1993, 115, 332-341.

[26] M. Sivakumar, A. B. Pandit, Ultrason. Sonochem. 2001, 8, 233-240.

[27] L. H. Thompson, L. K. Doraiswamy, Ind. Eng. Chem. Res. 1999, 38, 1215-1249.

[28] This clustering shields the inner bubbles from the transmission of acoustic energy. Usually a larger number of bubbles will be generated, followed by the significant dampening of ultrasound energy.

[29] A. Henglein, M. Gutierrez, J Phys. Chem. 1993, 97, 158-162.

[30] J. P. Lorimer, T. J. Mason, Chem. Soc. Rev. 1987, 16, 239-274.

[31] D. J. Laser, J. G. Santiago, J Micromech. Microeng. 2004, 14, R35-R64.

[32] E. Sayar, B. Farouk, Microfluid. Nanofluid. 2015, 18, 433-445.

[33] E. Sayar, B. Farouk, Sens. Actuators A Phys. 2011, 171, 317-323.

[34] M. S. Weinberg, C. E. Dube, A. Petrovich, A. M. Zapata, J Microelectromech. Syst. 2003, 12, 567-576.
[35] J. W. S. Rayleigh, The Theory of Sound, 2nd ed. Vol. 1, Dover Publications, New York, 1945, pp. 312-337.

[36] R. R. Boullosa, A. O. Santillan, Acta Acustica united with Acustica 2004 90, 277-284.

[37] H. Tian, T. L. Ren, D. Xie, Y. F. Wang, C. J. Zhou, T. T. Feng, D. Fu, Y. Yang, P. G. Peng, L. G. Wang, L. T. Liu, ACS Nano 2011, 5, 48784885.

[38] D. Foresti, G. Sambatakakis, S. Botta, D. Poulikakos, Scientific Reports 2013, 3, 3176-3176

[39] W. J. Xie, B. Wei, Phys. Rev. E 2002, 66, 026605-1-11.

[40] Z. Y. Hong, W. J. Xie, B. Wei, J Appl. Phys. 2010, 107, 014901-4.

[41] F. Parvizian, M. Rahimi, S. M. Hosseini, S. S. Madaeni, A. A. Alsairafi, Desalination 2012, 286, 155-165.

[42] P. Dlugolęcki, Mass transport in reverse electrodialysis for sustainable energy generation, Ph.D. Thesis, 2009, University of Twente.

[43] Y. K. Lee, C. Shih, P. Tabelin, C. M. Ho, J Fluid Mech. 2007, 575, 425448.

[44] X. Mao, J. R. Waldeisen, T. J. Huang, Lab Chip 2007, 7, 1260-1262.

[45] D. Erickson, D. Li, Langmuir 2002, 18, 1883-1892.

[46] J. H. Tsai, L. Lin L Sens. Actuators A 2002, 97, 665-671.

[47] A. N. Hellman, K. R. Rau, H. H. Yoon, S. Bae, J. F. Palmer, K. S. Phillips, N. L. Allbritton, V. Venugopalan, Anal. Chem. 2007, 79, 44844492.

[48] P. Marmottant, S. Hilgenfeldt, Proc. Nat. Acad. Sci. USA 2004, 101, 9523-9527.

[49] H. S. Seo, Y. H. Kim, J. Acoust. Soc. Am. 2005, 118, 92-103.

[50] V. V. Nikonenko, N. D. Pismenskaya, E. I. Belova, P. Sistat, P. Huguet, G. Pourcelly, C. Larchet, Adv. Colloid Interface Sci. 2010, 160, 101-123.

[51] S. M. Rubinstein, G. Manukyan, A. Staicu, I. Rubinstein, B. Zaltzman, R. G. H. Lammertink, F. Mugele, M. Wessling, Phys. Rev. Lett. 2008, 101, 236101-1-4.

[52] a) Y. X. Jiang, J. Dodds, D. Leclerc, M. Lenoel, J. Membr. Sci. 1995, 105, 23-30; b) J. Lindau, A. S. Jonsson, J. Membr. Sci. 1999, 160, 6576.

[53] J. A. Roberts, P. M. Sutton, P. N. Mishra, Int. Biodeter. Biodegr. 2000, 46, 37-42.

[54] Z. Wang, J. Ma, C. Y. Tang, K. Kimurc, Q. Wang, X. Han, J Membr. Sci. 2014, 468, 276-307.

[55] P. Le Clech, V. Chen, T. A. G. Fane, J. Membr. Sci. 2006, 284, 17-53.

[56] S. Judd, The MBR Book: Principles and Applications of Membrane Bioreactors for water and wastewater treatment, 2nd ed. (Ed.: S. Judd, C. Judd ), Elsevier: Butterworth-Heinemann, 2011, pp. 536.

[57] P. van den Brink, F. Vergeldt, H. Van As, A. Zwijnenburg, H. Temmink, M. C. M. van Loosdrecht, J Membr. Sci. 2013, 429, 259-267.

[58] H. C. Flemming, J. Wingender, Nat. Rev. Microbiol. 2010, 8, 623-633.

[59] a) S. Muthukumaran, S. E. Kentish., M. Ashokkumar, G. W. Stevens, J Membr. Sci. 2005, 258, 106-114; b) N. Ahner, D. Gottschlich., S. Narang., D. Roberts., S. Sharma, S. Ventura, Separ. Sci. Technol. 1993, 28, 895-908; c) X. Chai, T. Kobayashi, N. Fujii, J Membr. Sci. 1998, 148, 129-135; d) A. Simon, N. Gondrexon., S. Taha., J. Gabon G. Dorange, Separ. Sci. Technol. 2000, 35, 2619-2637.

[60] a) D. Chen, L. K. Weavers, H. W. Walker, Ultrason. Sonochem. 2006, 13, 379-387; b) M. Shibata, T. Kobayashi, N. Fujii, J Appl. Polym. Sci. 2000, 75, 1546-1553.

[61] S. Muthukumaran, S. E. Kentish, G. W. Stevens, M. Ashokkumar, 2006 22, 155-194

[62] T. L. Geers, M. Hasheminejad, J Acoust. Soc. Am. 1991, 90, 32383247.

[63] L. Rozenberg, "On the Physics of Ultrasonic Cleaning," Ultrasonic News 1960, 4, 16-20.

[64] J. M. Valverde, Eur. Phys. J. E 2015, 38, 66-1-13.

[65] L. D. Rozenberg, High-Intensity Ultrasonic Fields, Ultrasonic Technology, (Ed.: L. Rozenberg), Springer Science \& Business Media, 2013, pp. 429

[66] W. L. Nyborg, J. Acoust. Soc. Am., 1958, 30, 329-339 
[67] A. M. Ghabrial, E. G. Richardson, Acustica 1955, 5, 28-34

[68] a) H. Schlichting, Boundary Layer Theory, McGraw-Hill Co., New York, 1979, pp. 647-652; b) C. Wang, J Acoust. Soc. Am. 1982, 71, 350-584 c) A. Ghabrial, E. Richardson, Acustica 1955, 5, 28-34

[69] S. Luther, R. Mettin, P. Koch, W. Lauterborn, Ultrason. Sonochem. 2001, 8, 159-162.

[70] S. A. Elder, J. Acoust. Soc. Am. 1959, 31, 54-64

[71] A. Busnaina, I. Kashkoush, Chem. Eng. Commun. 1993, 125, 47-61.

[72] A. Busnaina, J. Taylor, I. Kashkoush, J. Adhesion Sci. Technol. 1993, 7, $441-455$.

[73] a) W. Nyborg, J Acoust Soc. Am. 1967, 42, 947-52; b) W. Nyborg Ultrasound Med. Biol. 2001, 27, 301-333.

[74] P. Marmottant, J. Raven, H. Garneniers, J. Bomer, S. Hilgenfeldt, J Fluid Mech. 2006, 568, 109-118.

[75] C. Chindam, N. Nama, M. I. Lapsley, F. Costanzo, T. J. Huang, J Appl. Phys. 2013, 114, 194503-1-7.

[76] R. P. Jiang, X. Q. Li, M. Zhang, Met. Mater. Int. 2015, 21, 104-108.

[77] a) G. I. Eskin, Ultrason. Sonochem. 2003, 10, 297-301; b) A. Das, H. R. Kotadia, Mater. Chem. Phys. 2011, 125, 853-859.

[78] H. Friedman, S. Reich, R. Popovitz Biro, Ultrason. Sonochem. 2013, 20 432-444.

[79] M. Qian, A. Ramirez, A. Das, J. Cryst. Growth. 2009, 14, 3708-3715.

[80] Z. Xu, L. Ma, J. Yan, W. Chen, S. Yang, Mater. Chem. Phys. 2014, 148 824-832.

[81] Q. Han, Metall Mater. Trans. 2015, 46B, 1603-1614.

[82] H. C. Flemming, J. Wingender, Nat. Rev. Microbiol. 2010, 8, 623-633.

[83] D. Or, S. Phutane, A. Dechesne, Vadose Zone J. 2007, 6, 298-305.

[84] H. C. Flemming, A. Leis, Encyclopedia of Environmental Microbiology (Ed.: G. Bitton), Wiley, New York, 2002, pp. 2958-2967.

[85] E. D. van Hullebusch, M. H. Zandvoord, P. N. L. Lens, Rev. Environ. Sci. Biotechnol. 2004, 2, 9-33.

[86] S. Wuertz, R. Spaeth, A. Hinderberger, T. Grieba, H. C. Flemming, P. A Wilderer, Water Sci. Technol. 2001, 43, 25-34.

[87] J. Schmitt, D. Nivens, D. C. White, H. C. Flemming, Water Sci. Technol. 1995, 32, 149-155.

[88] M. M. Klausen, T. R. Thomsen, J. L. Nielsen, L. H. Mikkelsen, P. H. Nielsen, FEMS Microbiol. Ecol. 2004, 50, 123-132.

[89] P. Stoodley, R. Cargo, C. J. Rupp, S. Wilson, I. Klapper, J. Ind. Microbiol. Biotechnol. 2003, 29, 361-367.

[90] C. J. Rupp, C. A. Fux, P. Stoodley, Appl. Environ. Microbiol. 2005, 71, 2175-2178.

[100] V. Körstgens, H. C. Flemming, J. Wingender, W. Borchard, Water Sci. Technol. 2001, 43, 49-57.

[101] T. Uemura, M. Henmi, Thin-film composite membranes for reverse osmosis, Advanced Membrane Technology and Applications, (Ed.: N. N. Li., A. G. Fane, W. S. W. Ho, T. Matsuura ), John Wiley and Sons Inc. New Jersey, 2008, pp. 3-20.

[102] S. T. V. Sim, S. R. Suwarno, T. H. Chong, W. B. Krantz, A. G. Fane, J Membr. Sci. 2013, 428, 24-37.

[103] A. Drews, J Membr. Sci. 2010, 363, 1-28

[104] W. N. Lee, I. S. Chang, B. K. Hwang, P. K. Park, C. H. Lee, X. Huang, Process. Biochem. 2007, 42, 655-661.

[105] K. G. Song, Y. Kim, K. H. Ahn, Desalination 2008, 221, 467-474.

[106] S. R. Chae, S. Wang, Z. D. Hendren, M. R. Wiesner, Y. Watanabe, C. K. Gunsch, J. Membr. Sci. 2009, 329, 68-74.

[107] Kenneth, J.H., J. C. Gerald., M. N. Steven, and J. K. Edward, Guide to Acid, Alkaline, Emulsion, and Ultrasonic Cleaning. 1997, The Materials Information Society, USA

[108] S. Kentish, H. Feng, Annu. Rev. Food Sci. Technol. 2014, 5, 263-84.

[109] I. Masselin, X. Chasseray, L. Durand-Bourlier, J.-M. Lainé, P.-Y. Syzaret, D. Lemordant, J. Membr. Sci. 2001, 181, 213-220.

[110] M. Band, M. Gutman, V. Faerman, E. Korngold, J. Kost, P.J. Plath, V. Gantar, Desalination 1997, 109, 303-313.

[111] T. Kokugan, T. Kaseno, S. Fujiwara, M. Shimizu, Membrane 1995, 20 213-223.
[112] G. Schulz, Desalination 1988, 68, 191-202.

[113] G. León, G. Martínez, M. A. Guzmán, J. I. Moreno, B. Miguel, J. A Fernández-López, Ultrason. Sonochem. 2013, 20, 650-654.

[114] R.-S. Juang, K.-H. Lin, Colloids and Surfaces A: Physicochem. Eng. Aspects 2004, 238, 43-49.

[115] a) C. del Cerro, D. Boey, Chem. Ind. 1988, 21, 681-687.

[116] T. Largman, S. Sifniades, Hydrometallurgy 1978, 3, 153-162.

[117] M. Chiha, O. Hamdaoui, F. Ahmedchekkat, C. Pétrier, Ultrason Sonochem. 2010, 17, 318-325

[118] D. Radziuk, H. Moehwald, Phys. Chem. Chem. Phys. 2015, DOI: 10.1039/c5cp05142h

[119] M. P. Thien, T. A. Hatton, Separ. Sci. Technol. 1988, 23, 819-653.

[120] R. R. Mohan, N. N. Li, Biorechnol. Bioeng. 1975, 17, 1137-1156.

[121] a) P. Stroeve, P. Varansi, J. Colloid Interface Sci. 1984, 99, 360-373; b) T. P. Martin, G. A. Davies, Hydrometallurgy 1977, 2, 315-334.

[122] S. Kiani, S. M. Mousavi, Ultrason. Sonochem. 2013, 20, 73-377.

[123] a) In cleaning of a silicon wafer, which is fouled by polystyrene latex particles, ultrasound $(40 \mathrm{kHz})$ effectively removes species of $0.3 \mu \mathrm{m}$ or larger. Both polymeric and ceramic membranes can be effectively cleaned by ultrasound; b) X. Chai, T. Kobayashi, N. Fujii, Sep. Purif. Technol. 1999, 15, 139-146.

[124] J. R. Thompson, Nondestructive vibratory cleaning system for reverse osmosis and ultrafiltration membranes, US Patent 4, 253, 962, 1981.

[125] A. Simon, L Penpenic., N. Gondrexon., S. Taha, G. Dorange, Ultrason. Sonochem. 2000, 7, 183-186.

[126] a) C. A. Romero, R. H. Davis, J. Membrane Sci. 1988, 39, 157-185; b) C. A. Romero, R. H. Davis, Chem. Eng. Sci. 1990, 45, 13-25.

[127] J. A. Schonberg, E. J. Hinch, J. Fluid Mech. 1989, 203, 517-524.

[128] H. Kyllonen, P. Pirkonen, M. Nystrom, J. Nuortila-Jokinen, A. Gronroos, Ultrason. Sonochem. 2006, 13, 295-302.

[129] F. Petersson, A. Nilsson, C. Holm, H. Jonsson, T. Laurell, Lab Chip 2005, 5, 20-22.

[130] A. H. J. Yang, H. T. Soh, Anal. Chem. 2012, 84, 10756-10762.

[131] T. Laurell, F. Petersson, A. Nilsson, Chem. Soc. Rev. 2007, 36, 492506.

[132] R. H. Liu, J. Yang, M. Z. Pindera, M. Athavale, P. Grodzinski, Lab Chip 2002, 2, 151-157.

[133] Y. Xie, C. Chindam, N. Nama, S. Yang, M. Lu, Y. Zhao, J. D. Mai, F. Costanzo, T. J. Huang, Scientific Reports 2015, 5, 12572-1-9.

[134] D. Mark, S. Haeberle, G. Roth, F. von Stettenz, R. Zengerlez, Chem. Soc. Rev. 2010, 39, 1153-1182.

[135] M. V. Patel, I. A. Nanayakkara, M. G. Simon, A. P. Lee, Lab Chip 2014 14, 3860-3872.

[136] D. Ahmed, X. Mao, J. Shi, B. K. Juluri, T. J. Huang, Lab Chip 2009, 9 2738-2741.

[137] a) C. Wang, S. V. Jalikop, S. Hilgenfeldt, Biomicrofluidics 2012, 6 , 012801-1-11; b) C. Wang, S. V. Jalikop, S. Hilgenfeldt, Appl. Phys. Lett 2011, 99, 034101-1-3

[138] P. Rogers, A. Neild, Lab Chip 2011, 11, 3710-3715.

[139] C. Wang, B. Rallabandi, S. Hilgenfeldt, Phys. Fluids 2013, 25, 022002 22011-220116.

[140] P. Marmottant, S. Hilgenfeldt, Nature 2003, 423, 153-156.

[141] a) N. Pamme, A. Manz, Anal. Chem. 2004, 76, 7250-7256; b) N Pamme, C. Wilhelm, Lab Chip 2006, 6, 974-980.

[142] U. Kim, C. W. Shu, K. Y. Dane, P. S. Daugherty, J. Y. J. Wang, H. T. Soh, Proc. Natl. Acad. Sci. U. S. A. 2007, 104, 20708-20712.

[143] M. Yamada, M. Seki, Anal. Chem. 2006, 78, 1357-1362.

[144] S. K. Cho, H. Moon, BioChip J 2008, 2, 79-96.

[145] Y. Zhao, S. K. Cho, Lab Chip 2007, 7, 273-280.

[146] W. Longsine-Parker, H. Wang, C. Koo, J. Kim, B. Kim, A. Jayaramane, A. Han, Lab Chip 2013, 13, 2144-2152.

[147] J. Voldman, Annual Rev. Biomed. Eng. 2006, 8, 425-454.

[148] J. Lee, H. Moon, J. Fowler, T. Schoellhammer, C. J. Kim, Sens. Actuators A 2002, 95, 259-268.

[149] F. Mugele, J. C. Baret, J. Phys.: Condens. Matter 2005, 17, R705R774 